\title{
Synthesis of Small Fluorescent Molecules and Evaluation of Photophysical Properties
}

Futa Ogawa, ${ }^{\dagger}$ Yukiko Karuo, ${ }^{\dagger}$ Ryuji Yamazawa ${ }^{\dagger}$ Kanae Miyanaga, ${ }^{\ddagger}$ Kazushige Hori, ${ }^{\ddagger}$ Keita Tani, ${ }^{\ddagger}$ Kengo Yamada, ${ }^{\mathbb{P}}$ Yuki Saito, ${ }^{\mathbb{P}}$ Kazumasa Funabiki, ${ }^{\mathbb{P}}$ Atsushi Tarui, ${ }^{\dagger}$ Kazuyuki Sato, ${ }^{\dagger}$ Kiyoshi Ito, $^{\dagger}$ Kentaro Kawai, ${ }^{\dagger}$ and Masaaki Omote ${ }^{\dagger *}$

\section{Supporting Information}

Computational calculation data S2-S19

FWHM of $3 \mathrm{a}$ and $3 \mathrm{c}-\mathrm{g}$. S20

Measurement of enzyme activity using H-Ala-3f as a substrate. S20

${ }^{1} \mathrm{H}$ and ${ }^{13} \mathrm{C}$ NMR Charts. S21-S28 
Computational Details. All calculations were performed using computational chemistry software package Gaussian 16 ver. B. $01^{1}$ using computational resource provided by Fujitsu PRIMERGY CX400 at Information and Communications Headquarters in Nagoya University.

\section{a) Ground State Details.}

Ground state geometries of 3a-3g were computed at RB3LYP/6-31G(d,p) level of theory. At the optimized structures, no imaginary frequency was found through the frequency analysis. All coordinates are reported as XYZ Cartesian coordinates. And computed E (RB3LYP) and sum of zero-point and thermal correction energies of optimized structures are shown.

3a

$\mathrm{E}(\mathrm{RB} 3 \mathrm{LYP})=-702.057413$ a.u.

Sum of electronic and thermal Energies $=-701.889801$ a.u.

Imaginary Frequency $=0$

Table S1. Cartesian coordinates of the optimized 3a.

\begin{tabular}{crrr}
\hline & \multicolumn{3}{c}{ Coordinates (Angstroms) } \\
Atom & \multicolumn{1}{c}{$\mathrm{X}$} & \multicolumn{1}{c}{$\mathrm{Y}$} & \multicolumn{1}{c}{$\mathrm{Z}$} \\
\hline $\mathrm{C}$ & -1.302587 & 0.296696 & -0.178903 \\
$\mathrm{H}$ & -1.135813 & 1.262569 & -0.641161 \\
$\mathrm{C}$ & -0.310731 & -0.534105 & 0.184597 \\
$\mathrm{H}$ & -0.611245 & -1.509123 & 0.563213 \\
$\mathrm{C}$ & -2.741576 & -0.074322 & -0.051169 \\
$\mathrm{~F}$ & -3.409253 & 0.81211 & 0.731417 \\
$\mathrm{~F}$ & -3.363575 & -0.06148 & -1.255068 \\
$\mathrm{~F}$ & -2.931427 & -1.299724 & 0.485802 \\
$\mathrm{C}$ & 1.138025 & -0.343567 & 0.097905 \\
$\mathrm{C}$ & 1.78638 & 0.920585 & 0.0353 \\
$\mathrm{C}$ & 1.938209 & -1.50268 & 0.082948 \\
$\mathrm{C}$ & 3.185277 & 0.961545 & -0.096029 \\
$\mathrm{C}$ & 3.319188 & -1.451404 & -0.049823 \\
$\mathrm{H}$ & 1.441513 & -2.466475 & 0.159551 \\
$\mathrm{C}$ & 3.940482 & -0.203284 & -0.149681 \\
$\mathrm{H}$ & 3.676574 & 1.930065 & -0.150192 \\
$\mathrm{H}$ & 3.903539 & -2.365136 & -0.072432 \\
$\mathrm{H}$ & 5.019541 & -0.135223 & -0.25276
\end{tabular}




$\begin{array}{lllr}\mathrm{N} & 1.067552 & 2.114207 & 0.078235 \\ \mathrm{H} & 0.224126 & 2.083868 & 0.637484 \\ \mathrm{H} & 1.631186 & 2.925078 & 0.29843\end{array}$

\section{3b}

$\mathrm{E}(\mathrm{RB} 3 \mathrm{LYP})=-702.059965$ a.u.

Sum of electronic and thermal Energies $=-701.892786$ a.u.

Imaginary Frequency $=0$

Table S2. Cartesian coordinates of the optimized 3b.

\begin{tabular}{crrr}
\hline & \multicolumn{3}{c}{ Coordinates (Angstroms) } \\
Atom & \multicolumn{1}{c}{$\mathrm{X}$} & \multicolumn{1}{c}{$\mathrm{Y}$} & \multicolumn{1}{c}{$\mathrm{Z}$} \\
\hline $\mathrm{C}$ & 1.634034 & 1.331993 & 0.003333 \\
$\mathrm{C}$ & 3.013006 & 1.173791 & 0.003847 \\
$\mathrm{C}$ & 3.581625 & -0.112016 & 0.005282 \\
$\mathrm{C}$ & 2.71563 & -1.224291 & 0.006147 \\
$\mathrm{C}$ & 1.341029 & -1.05231 & 0.005088 \\
$\mathrm{C}$ & 0.759567 & 0.230452 & 0.003425 \\
$\mathrm{H}$ & 1.218112 & 2.336325 & 0.000208 \\
$\mathrm{H}$ & 3.660371 & 2.04686 & 0.006834 \\
$\mathrm{H}$ & 3.13551 & -2.226959 & 0.011321 \\
$\mathrm{H}$ & 0.707424 & -1.934054 & 0.002741 \\
$\mathrm{~N}$ & 4.95713 & -0.285437 & 0.059699 \\
$\mathrm{H}$ & 5.308099 & -1.176696 & -0.260106 \\
$\mathrm{H}$ & 5.518067 & 0.493361 & -0.254421 \\
$\mathrm{C}$ & -0.682138 & 0.467217 & -0.000417 \\
$\mathrm{H}$ & -0.978007 & 1.514142 & -0.005801 \\
$\mathrm{C}$ & -1.66442 & -0.445925 & 0.00359 \\
$\mathrm{H}$ & -1.483595 & -1.516038 & 0.011155 \\
$\mathrm{C}$ & -3.107727 & -0.081096 & -0.001774 \\
$\mathrm{~F}$ & -3.317514 & 1.254498 & -0.008854 \\
$\mathrm{~F}$ & -3.745662 & -0.591591 & -1.085921 \\
$\mathrm{~F}$ & -3.751216 & -0.581324 & 1.083559
\end{tabular}


$3 \mathrm{c}$

$E(R B 3 L Y P)=-794.300093$ a.u.

Sum of electronic and thermal Energies $=-794.132075$ a.u.

Imaginary Frequency $=0$

Table S3. Cartesian coordinates of the optimized 3c.

\begin{tabular}{crrr}
\hline & \multicolumn{3}{c}{ Coordinates (Angstroms) } \\
Atom & \multicolumn{1}{c}{$\mathrm{X}$} & \multicolumn{1}{c}{$\mathrm{Y}$} & \multicolumn{1}{c}{$\mathrm{Z}$} \\
\hline $\mathrm{C}$ & 1.893357 & 1.893357 & 1.893357 \\
$\mathrm{H}$ & 1.930541 & 1.930541 & 1.930541 \\
$\mathrm{C}$ & 0.75502 & 0.75502 & 0.75502 \\
$\mathrm{H}$ & 0.840942 & 0.840942 & 0.840942 \\
$\mathrm{C}$ & 3.223545 & 3.223545 & 3.223545 \\
$\mathrm{~F}$ & 4.07411 & 4.07411 & 4.07411 \\
$\mathrm{~F}$ & 3.806936 & 3.806936 & 3.806936 \\
$\mathrm{~F}$ & 3.151885 & 3.151885 & 3.151885 \\
$\mathrm{C}$ & -0.618899 & -0.618899 & -0.618899 \\
$\mathrm{C}$ & -0.970572 & -0.970572 & -0.970572 \\
$\mathrm{C}$ & -1.648679 & -1.648679 & -1.648679 \\
$\mathrm{C}$ & -2.327982 & -2.327982 & -2.327982 \\
$\mathrm{C}$ & -2.993665 & -2.993665 & -2.993665 \\
$\mathrm{H}$ & -1.389981 & -1.389981 & -1.389981 \\
$\mathrm{C}$ & -3.323283 & -3.323283 & -3.323283 \\
$\mathrm{H}$ & -2.591832 & -2.591832 & -2.591832 \\
$\mathrm{H}$ & -4.361278 & -4.361278 & -4.361278 \\
$\mathrm{~N}$ & -0.014288 & -0.014288 & -0.014288 \\
$\mathrm{H}$ & 0.837205 & 0.837205 & 0.837205 \\
$\mathrm{H}$ & -0.363486 & -0.363486 & -0.363486 \\
$\mathrm{C}$ & -4.015103 & -4.015103 & -4.015103 \\
$\mathrm{~N}$ & -4.8487 & -2.250925 & 0.040126
\end{tabular}


3d

$E(R B 3 L Y P)=-741.378670$ a.u.

Sum of electronic and thermal Energies $=-741.181803$ a.u.

Imaginary Frequency $=0$

Table S4. Cartesian coordinates of the optimized 3d.

\begin{tabular}{crrr}
\hline & \multicolumn{3}{c}{ Coordinates (Angstroms) } \\
Atom & $\mathrm{X}$ & $\mathrm{Y}$ & \multicolumn{1}{c}{$\mathrm{Z}$} \\
\hline $\mathrm{C}$ & 1.741484 & 0.296822 & 0.178787 \\
$\mathrm{H}$ & 1.574825 & 1.266578 & 0.632745 \\
$\mathrm{C}$ & 0.748933 & -0.530874 & -0.192255 \\
$\mathrm{H}$ & 1.049434 & -1.510559 & -0.558847 \\
$\mathrm{C}$ & 3.179186 & -0.083862 & 0.073653 \\
$\mathrm{~F}$ & 3.864318 & 0.791404 & -0.707122 \\
$\mathrm{~F}$ & 3.786152 & -0.064859 & 1.28558 \\
$\mathrm{~F}$ & 3.369078 & -1.315085 & -0.450442 \\
$\mathrm{C}$ & -0.698118 & -0.331505 & -0.130766 \\
$\mathrm{C}$ & -1.3447 & 0.932357 & -0.078508 \\
$\mathrm{C}$ & -1.511541 & -1.481778 & -0.134331 \\
$\mathrm{C}$ & -2.745418 & 0.979591 & 0.024502 \\
$\mathrm{C}$ & -2.891919 & -1.418997 & -0.029123 \\
$\mathrm{H}$ & -1.025107 & -2.451268 & -0.206202 \\
$\mathrm{C}$ & -3.529546 & -0.171371 & 0.065034 \\
$\mathrm{H}$ & -3.227765 & 1.954145 & 0.06752 \\
$\mathrm{H}$ & -3.479981 & -2.332015 & -0.023459 \\
$\mathrm{~N}$ & -0.622894 & 2.12461 & -0.107675 \\
$\mathrm{H}$ & 0.23105 & 2.091583 & -0.650629 \\
$\mathrm{H}$ & -1.180422 & 2.936518 & -0.339293 \\
$\mathrm{C}$ & -5.029144 & -0.080899 & 0.209503 \\
$\mathrm{H}$ & -5.397273 & 0.923411 & -0.017206 \\
$\mathrm{H}$ & -5.536136 & -0.787575 & -0.455354 \\
$\mathrm{H}$ & -5.339592 & -0.323144 & 1.233317
\end{tabular}


$3 \mathbf{e}$

$E(R B 3 L Y P)=-816.584081$ a.u.

Sum of electronic and thermal Energies $=-816.381240$ a.u.

Imaginary Frequency $=0$

Table S5. Cartesian coordinates of the optimized $3 \mathbf{e}$.

\begin{tabular}{crrr}
\hline & \multicolumn{3}{c}{ Coordinates (Angstroms) } \\
Atom & \multicolumn{1}{c}{$\mathrm{X}$} & $\mathrm{Y}$ & $\mathrm{Z}$ \\
\hline $\mathrm{C}$ & 2.156858 & 0.275442 & 0.181168 \\
$\mathrm{H}$ & 2.04443 & 1.251555 & 0.637778 \\
$\mathrm{C}$ & 1.12014 & -0.494113 & -0.196977 \\
$\mathrm{H}$ & 1.369175 & -1.488079 & -0.563849 \\
$\mathrm{C}$ & 3.569989 & -0.186818 & 0.080633 \\
$\mathrm{~F}$ & 4.308682 & 0.64662 & -0.69783 \\
$\mathrm{~F}$ & 4.174567 & -0.203036 & 1.29431 \\
$\mathrm{~F}$ & 3.69099 & -1.427546 & -0.442892 \\
$\mathrm{C}$ & -0.312843 & -0.218856 & -0.147407 \\
$\mathrm{C}$ & -0.897432 & 1.080573 & -0.078273 \\
$\mathrm{C}$ & -1.185089 & -1.319993 & -0.173937 \\
$\mathrm{C}$ & -2.286259 & 1.203771 & 0.017639 \\
$\mathrm{C}$ & -2.56595 & -1.203378 & -0.07447 \\
$\mathrm{H}$ & -0.750606 & -2.312913 & -0.255524 \\
$\mathrm{C}$ & -3.11798 & 0.081258 & 0.034453 \\
$\mathrm{H}$ & -2.747395 & 2.185285 & 0.072529 \\
$\mathrm{H}$ & -3.186426 & -2.089985 & -0.084735 \\
$\mathrm{~N}$ & -0.108963 & 2.229151 & -0.085361 \\
$\mathrm{H}$ & 0.741584 & 2.155961 & -0.629295 \\
$\mathrm{H}$ & -0.618049 & 3.07687 & -0.298898 \\
$\mathrm{C}$ & -5.354451 & -0.752271 & 0.150424 \\
$\mathrm{H}$ & -6.3502 & -0.317556 & 0.246259 \\
$\mathrm{H}$ & -5.300841 & -1.330346 & -0.780371 \\
$\mathrm{H}$ & -5.168386 & -1.420306 & 1.000329 \\
$\mathrm{O}$ & -4.450075 & 0.343428 & 0.138936
\end{tabular}


$\mathrm{E}(\mathrm{RB} 3 \mathrm{LYP})=-794.298258$ a.u.

Sum of electronic and thermal Energies $=-794.130305$ a.u.

Imaginary Frequency $=0$

Table S6. Cartesian coordinates of the optimized $\mathbf{3 f}$.

\begin{tabular}{crrr}
\hline & \multicolumn{3}{c}{ Coordinates (Angstroms) } \\
Atom & \multicolumn{1}{c}{$\mathrm{X}$} & $\mathrm{Y}$ & \multicolumn{1}{c}{$\mathrm{Z}$} \\
\hline $\mathrm{C}$ & 1.96966 & 0.286488 & 0.193153 \\
$\mathrm{H}$ & 1.790626 & 1.239717 & 0.677024 \\
$\mathrm{C}$ & 0.98887 & -0.530985 & -0.22258 \\
$\mathrm{H}$ & 1.294541 & -1.495689 & -0.62199 \\
$\mathrm{C}$ & 3.413503 & -0.083982 & 0.093932 \\
$\mathrm{~F}$ & 4.097786 & 0.826449 & -0.642 \\
$\mathrm{~F}$ & 3.996117 & -0.109478 & 1.314897 \\
$\mathrm{~F}$ & 3.613199 & -1.291667 & -0.475737 \\
$\mathrm{C}$ & -0.461655 & -0.333248 & -0.160456 \\
$\mathrm{C}$ & -1.102481 & 0.937002 & -0.108225 \\
$\mathrm{C}$ & -1.266688 & -1.487701 & -0.162424 \\
$\mathrm{C}$ & -2.500131 & 0.989157 & 0.000078 \\
$\mathrm{C}$ & -2.647206 & -1.437177 & -0.052903 \\
$\mathrm{H}$ & -0.77601 & -2.454265 & -0.232019 \\
$\mathrm{C}$ & -3.266466 & -0.179468 & 0.03978 \\
$\mathrm{H}$ & -2.996054 & 1.953672 & 0.048152 \\
$\mathrm{H}$ & -3.242537 & -2.342247 & -0.040379 \\
$\mathrm{~N}$ & -0.378149 & 2.122311 & -0.137017 \\
$\mathrm{H}$ & 0.484228 & 2.090627 & -0.665527 \\
$\mathrm{H}$ & -0.925525 & 2.945326 & -0.351105 \\
$\mathrm{C}$ & -4.693067 & -0.085305 & 0.154342 \\
$\mathrm{~N}$ & -5.850312 & -0.009962 & 0.246049
\end{tabular}


$\mathrm{E}(\mathrm{RB} 3 \mathrm{LYP})=-929.939492$ a.u.

Sum of electronic and thermal Energies $=-929.724404$ a.u.

Imaginary Frequency $=0$

Table S7. Cartesian coordinates of the optimized 3g.

\begin{tabular}{crrr}
\hline & \multicolumn{3}{c}{ Coordinates (Angstroms) } \\
Atom & $\mathrm{X}$ & $\mathrm{Y}$ & $\mathrm{Z}$ \\
\hline $\mathrm{C}$ & 2.837679 & 0.251636 & 0.191156 \\
$\mathrm{H}$ & 2.697642 & 1.217524 & 0.662217 \\
$\mathrm{C}$ & 1.82374 & -0.524024 & -0.22689 \\
$\mathrm{H}$ & 2.08993 & -1.50611 & -0.612287 \\
$\mathrm{C}$ & 4.262372 & -0.187276 & 0.114014 \\
$\mathrm{~F}$ & 5.000563 & 0.680169 & -0.623219 \\
$\mathrm{~F}$ & 4.831138 & -0.226333 & 1.342315 \\
$\mathrm{~F}$ & 4.41353 & -1.410215 & -0.439433 \\
$\mathrm{C}$ & 0.383671 & -0.261479 & -0.186476 \\
$\mathrm{C}$ & -0.202746 & 1.033797 & -0.127748 \\
$\mathrm{C}$ & -0.472792 & -1.379497 & -0.214317 \\
$\mathrm{C}$ & -1.597488 & 1.141663 & -0.041431 \\
$\mathrm{C}$ & -1.851617 & -1.265745 & -0.125389 \\
$\mathrm{H}$ & -0.023107 & -2.365965 & -0.28776 \\
$\mathrm{C}$ & -2.416679 & 0.013982 & -0.028195 \\
$\mathrm{H}$ & -2.066832 & 2.119349 & 0.011193 \\
$\mathrm{H}$ & -2.485365 & -2.143299 & -0.133223 \\
$\mathrm{~N}$ & 0.573588 & 2.190483 & -0.128389 \\
$\mathrm{H}$ & 1.427476 & 2.1335 & -0.669123 \\
$\mathrm{H}$ & 0.054051 & 3.033405 & -0.335992 \\
$\mathrm{C}$ & -3.888214 & 0.23824 & 0.068257 \\
$\mathrm{O}$ & -4.415188 & 1.33185 & 0.137224 \\
$\mathrm{O}$ & -4.586543 & -0.919413 & 0.071518 \\
$\mathrm{C}$ & -6.012344 & -0.772776 & 0.163515 \\
$\mathrm{H}$ & -6.395446 & -0.198574 & -0.683551 \\
$\mathrm{H}$ & -6.413015 & -1.786055 & 0.152749 \\
$\mathrm{H}$ & -6.287191 & -0.260354 & 1.088623
\end{tabular}




\section{b) Excited State Calculation}

Using the optimized geometry of the ground state $\left(\mathrm{S}_{0}\right)$, single point time dependent density functional theory (TD-DFT) calculations were performed using the UB3LYP/6-31+G(d,p) level of theory. For the TD-DFT calculations, the polarizable continuum model (PCM) was employed to take into account the effect of the polarity of THF. The first excited states of 3a-3g are reported below. In calculating 100 kinds of transitions, dominant UV-vis absorption peaks with significant and relevant oscillator strengths (f value) ( $\mathrm{f} \geqq 0.1)$ are highlighted.

$\underline{\mathbf{3 a}}$

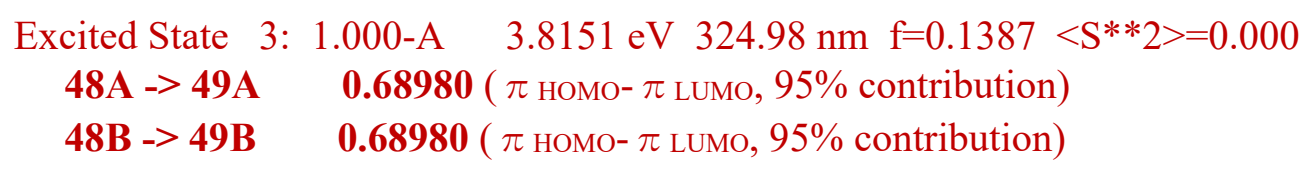

\begin{tabular}{|c|c|}
\hline Excited State & $1.000-\mathrm{A}$ \\
\hline $47 \mathrm{~A}->49 \mathrm{~A}$ & 0.55807 \\
\hline $48 \mathrm{~A}->49 \mathrm{~A}$ & 0.11915 \\
\hline $48 \mathrm{~A}->50 \mathrm{~A}$ & 0.39536 \\
\hline $47 \mathrm{~B}->49 \mathrm{~B}$ & 0.55807 \\
\hline $48 B->49 B$ & 0.11915 \\
\hline $48 \mathrm{~B}->50 \mathrm{~B}$ & 0.39536 \\
\hline
\end{tabular}

Excited State 9: 1.000-A
$47 \mathrm{~A} \rightarrow 49 \mathrm{~A} \quad-0.32192$
$48 \mathrm{~A} \rightarrow 50 \mathrm{~A} \quad 0.46067$
$48 \mathrm{~A} \rightarrow 51 \mathrm{~A} \quad 0.37847$
$48 \mathrm{~A} \rightarrow 54 \mathrm{~A} \quad-0.13255$
$47 \mathrm{~B}->49 \mathrm{~B} \quad-0.32192$
$48 \mathrm{~B}->50 \mathrm{~B} \quad 0.46067$
$48 \mathrm{~B}->51 \mathrm{~B} \quad 0.37847$
$48 \mathrm{~B}->54 \mathrm{~B} \quad-0.13255$

Excited State 10: $1.000-\mathrm{A} \quad 5.2625 \mathrm{eV} \quad 235.60 \mathrm{~nm} \mathrm{f}=0.1693<\mathrm{S} * * 2>=0.000$

$47 \mathrm{~A} \rightarrow$ 49A $\quad 0.22957$

$48 \mathrm{~A} \rightarrow 50 \mathrm{~A} \quad-0.26761$

$48 \mathrm{~A} \rightarrow 51 \mathrm{~A} \quad 0.57882$

$48 \mathrm{~A} \rightarrow 52 \mathrm{~A} \quad-0.11960$

$48 \mathrm{~A} \rightarrow 53 \mathrm{~A} \quad-0.10821$

$47 \mathrm{~B} \rightarrow 49 \mathrm{~B} \quad 0.22957$

$48 \mathrm{~B}->50 \mathrm{~B} \quad-0.26761$

$48 \mathrm{~B}->51 \mathrm{~B} \quad 0.57882$

$48 \mathrm{~B}->52 \mathrm{~B} \quad-0.11960$

$48 \mathrm{~B}->53 \mathrm{~B} \quad-0.10821$

Excited State 14: $1.000-\mathrm{A} \quad 5.7507 \mathrm{eV} \quad 215.60 \mathrm{~nm} \mathrm{f}=0.1766<\mathrm{S} * * 2>=0.000$
$45 \mathrm{~A} \rightarrow 49 \mathrm{~A}$
0.11311
$47 \mathrm{~A} \rightarrow 50 \mathrm{~A}$
0.41233

$4.7793 \mathrm{eV} 259.42 \mathrm{~nm} \mathrm{f}=0.1869<\mathrm{S} * * 2>=0.000$

$5.2077 \mathrm{eV} 238.08 \mathrm{~nm} \mathrm{f}=0.2947<\mathrm{S}^{* * 2}>=0.000$ 


$$
\begin{array}{lr}
48 \mathrm{~A}->50 \mathrm{~A} & -0.15725 \\
48 \mathrm{~A}->53 \mathrm{~A} & 0.48577 \\
48 \mathrm{~A}->54 \mathrm{~A} & -0.20039 \\
45 \mathrm{~B}->49 \mathrm{~B} & 0.11311 \\
47 \mathrm{~B}->50 \mathrm{~B} & 0.41233 \\
48 \mathrm{~B}->50 \mathrm{~B} & -0.15725 \\
48 \mathrm{~B}->53 \mathrm{~B} & 0.48577 \\
48 \mathrm{~B}->54 \mathrm{~B} & -0.20039
\end{array}
$$

Excited State 18: 1.000-A

$\begin{array}{lr}45 \mathrm{~A}->49 \mathrm{~A} & 0.15757 \\ 46 \mathrm{~A}->49 \mathrm{~A} & 0.23231 \\ 47 \mathrm{~A}->50 \mathrm{~A} & 0.43580 \\ 48 \mathrm{~A}->52 \mathrm{~A} & -0.10209 \\ 48 \mathrm{~A}->53 \mathrm{~A} & -0.25070 \\ 48 \mathrm{~A}->54 \mathrm{~A} & 0.33509 \\ 45 \mathrm{~B}->49 \mathrm{~B} & 0.15757 \\ 46 \mathrm{~B}->49 \mathrm{~B} & 0.23231 \\ 47 \mathrm{~B}->50 \mathrm{~B} & 0.43580 \\ 48 \mathrm{~B}->52 \mathrm{~B} & -0.10209 \\ 48 \mathrm{~B}->53 \mathrm{~B} & -0.25070 \\ 48 \mathrm{~B}->54 \mathrm{~B} & 0.33509\end{array}$

Excited State 34: $1.000-\mathrm{A} \quad 6.6825 \mathrm{eV} \quad 185.54 \mathrm{~nm} \mathrm{f}=0.1512<\mathrm{S} * * 2>=0.000$
$44 \mathrm{~A}->49 \mathrm{~A}$
$-0.12331$
$45 \mathrm{~A} \rightarrow 49 \mathrm{~A} \quad 0.45820$
$47 \mathrm{~A} \rightarrow 50 \mathrm{~A} \quad-0.14928$
$47 \mathrm{~A} \rightarrow 53 \mathrm{~A} \quad 0.39862$
$47 \mathrm{~A} \rightarrow 54 \mathrm{~A} \quad 0.14733$
$48 \mathrm{~A} \rightarrow 56 \mathrm{~A} \quad 0.10048$
$48 \mathrm{~A} \rightarrow 57 \mathrm{~A} \quad-0.11576$
$48 \mathrm{~A} \rightarrow 58 \mathrm{~A} \quad 0.10947$
$44 \mathrm{~B}->49 \mathrm{~B} \quad-0.12331$
$45 \mathrm{~B}->49 \mathrm{~B} \quad 0.45820$
$47 \mathrm{~B}->50 \mathrm{~B} \quad-0.14928$
$47 \mathrm{~B} \rightarrow 53 \mathrm{~B} \quad 0.39862$
$47 \mathrm{~B}->54 \mathrm{~B} \quad 0.14733$
$48 \mathrm{~B}->56 \mathrm{~B} \quad 0.10048$
$48 \mathrm{~B}->57 \mathrm{~B} \quad-0.11576$
$48 \mathrm{~B}->58 \mathrm{~B} \quad 0.10947$

$6.1126 \mathrm{eV} 202.83 \mathrm{~nm} \mathrm{f}=0.2557<\mathrm{S} * * 2>=0.000$

3b

Excited State 3: $1.000-\mathrm{A} \quad 4.0369 \mathrm{eV} \quad 307.13 \mathrm{~nm} \mathrm{f}=0.6408<\mathrm{S} * * 2>=0.000$
48A $\rightarrow$ 49A
$\mathbf{0 . 6 9 0 6 1}$ ( $\pi$ номо- $\pi$ LUMO, $95 \%$ contribution)
$48 \mathrm{~A} \rightarrow 50 \mathrm{~A}$
$-0.12134$
48B $\rightarrow$ 49B
$\mathbf{0 . 6 9 0 6 1}$ ( $\pi$ номо- $\pi$ LUMO, 95\% contribution)
$48 \mathrm{~B}->50 \mathrm{~B}$
$-0.12134$

Excited State 10: $1.000-\mathrm{A} \quad 5.2936 \mathrm{eV} \quad 234.22 \mathrm{~nm} \mathrm{f}=0.1925<\mathrm{S} * * 2>=0.000$ 


$$
\begin{array}{lr}
47 \mathrm{~A}->49 \mathrm{~A} & 0.62168 \\
48 \mathrm{~A}->50 \mathrm{~A} & -0.30734 \\
47 \mathrm{~B}->49 \mathrm{~B} & 0.62168 \\
48 \mathrm{~B}->50 \mathrm{~B} & -0.30734
\end{array}
$$

Excited State 18: 1.000-A

$$
\begin{array}{lr}
46 \mathrm{~A} \rightarrow 49 \mathrm{~A} & 0.49146 \\
47 \mathrm{~A}->50 \mathrm{~A} & -0.25745 \\
48 \mathrm{~A}->54 \mathrm{~A} & -0.31825 \\
48 \mathrm{~A}->55 \mathrm{~A} & -0.23479 \\
46 \mathrm{~B}->49 \mathrm{~B} & 0.49146 \\
47 \mathrm{~B}->50 \mathrm{~B} & -0.25745 \\
48 \mathrm{~B}->54 \mathrm{~B} & -0.31825 \\
48 \mathrm{~B}->55 \mathrm{~B} & -0.23479
\end{array}
$$

Excited State 28: 1.000-A
$46 \mathrm{~A} \rightarrow 49 \mathrm{~A}$
0.10400
$46 \mathrm{~A} \rightarrow 50 \mathrm{~A}$
$-0.39384$
$47 \mathrm{~A} \rightarrow 50 \mathrm{~A}$
0.48215
$47 \mathrm{~A} \rightarrow 51 \mathrm{~A}$
0.18806
$48 \mathrm{~A}->54 \mathrm{~A} \quad-0.13164$
$46 \mathrm{~B}->$ 49B $\quad 0.10400$
$46 \mathrm{~B}->50 \mathrm{~B} \quad-0.39384$
$47 \mathrm{~B}->50 \mathrm{~B} \quad 0.48215$
$47 \mathrm{~B}->51 \mathrm{~B} \quad 0.18806$
$48 \mathrm{~B}->54 \mathrm{~B} \quad-0.13164$

Excited State 31: 1.000-A $46 \mathrm{~A} \rightarrow$ 50A $\quad 0.30297$ $47 \mathrm{~A} \rightarrow 50 \mathrm{~A} \quad 0.26057$ $48 \mathrm{~A} \rightarrow 54 \mathrm{~A} \quad-0.10681$

$48 \mathrm{~A} \rightarrow 57 \mathrm{~A} \quad-0.11430$

$48 \mathrm{~A}->58 \mathrm{~A} \quad 0.51752$

$46 \mathrm{~B}->50 \mathrm{~B} \quad 0.30297$

$47 \mathrm{~B}->50 \mathrm{~B} \quad 0.26057$

$48 \mathrm{~B}->54 \mathrm{~B} \quad-0.10681$

$48 \mathrm{~B}->57 \mathrm{~B} \quad-0.11430$

$48 \mathrm{~B}->58 \mathrm{~B} \quad 0.51752$

Excited State 46: $1.000-\mathrm{A} \quad 7.1735 \mathrm{eV} \quad 172.84 \mathrm{~nm} \mathrm{f}=0.1401<\mathrm{S} * * 2>=0.000$
$47 \mathrm{~A} \rightarrow 54 \mathrm{~A}$
0.64802
$47 \mathrm{~A} \rightarrow 55 \mathrm{~A}$
$-0.12428$
$47 \mathrm{~B}->54 \mathrm{~B}$
0.64802
$47 \mathrm{~B}->55 \mathrm{~B}$
$-0.12428$

\section{$\underline{3 c}$}

Excited State 4: 1.000-A

$3.8717 \mathrm{eV} 320.23 \mathrm{~nm} \mathrm{f}=0.1195<\mathrm{S} * * 2>=0.000$

$6.0055 \mathrm{eV} 206.45 \mathrm{~nm} \mathrm{f}=0.1552<\mathrm{S} * * 2>=0.000$

$6.4966 \mathrm{eV} 190.84 \mathrm{~nm} \mathrm{f}=0.2943<\mathrm{S} * * 2>=0.000$

$6.6276 \mathrm{eV} 187.07 \mathrm{~nm} \mathrm{f}=0.1466<\mathrm{S} * * 2>=0.000$

$$
\begin{array}{ll}
53 \mathrm{~A}->55 \mathrm{~A} & -0.10913 \\
53 \mathrm{~A}->56 \mathrm{~A} & -0.10062
\end{array}
$$




\begin{tabular}{|c|c|}
\hline $54 \mathrm{~A}->55 \mathrm{~A}$ & $\mathbf{0 . 6 6 5 5 6}$ ( $\pi$ номо- $\pi$ LUMO, $89 \%$ contribution $)$ \\
\hline $54 \mathrm{~A}->56 \mathrm{~A}$ & -0.17923 \\
\hline $53 \mathrm{~B}->55 \mathrm{~B}$ & -0.10913 \\
\hline $53 \mathrm{~B}->56 \mathrm{~B}$ & -0.10062 \\
\hline $54 B->55 B$ & $\mathbf{0 . 6 6 5 5 6}(\pi$ нОмо- $\pi$ LUMO, $89 \%$ contribution $)$ \\
\hline $54 \mathrm{~B}->56 \mathrm{~B}$ & -0.17923 \\
\hline
\end{tabular}

Excited State 6: 1.000-A

$4.5323 \mathrm{eV} 273.56 \mathrm{~nm} \mathrm{f}=0.1645<\mathrm{S} * * 2>=0.000$

$\begin{array}{ll}53 \mathrm{~A}->55 \mathrm{~A} & 0.30314 \\ 54 \mathrm{~A}->55 \mathrm{~A} & 0.21393 \\ 54 \mathrm{~A}->56 \mathrm{~A} & 0.58962 \\ 53 \mathrm{~B}->55 \mathrm{~B} & 0.30314 \\ 54 \mathrm{~B}->55 \mathrm{~B} & 0.21393 \\ 54 \mathrm{~B}->56 \mathrm{~B} & 0.58962\end{array}$

Excited State 9: $1.000-\mathrm{A} \quad 5.1246 \mathrm{eV} \quad 241.94 \mathrm{~nm} \mathrm{f}=1.0950<\mathrm{S} * * 2>=0.000$

$53 \mathrm{~A} \rightarrow 55 \mathrm{~A} \quad 0.53744$

$53 \mathrm{~A} \rightarrow 56 \mathrm{~A} \quad 0.25669$

$54 \mathrm{~A} \rightarrow 56 \mathrm{~A} \quad-0.32605$

$54 \mathrm{~A} \rightarrow 57 \mathrm{~A} \quad-0.12816$

$53 \mathrm{~B}->55 \mathrm{~B} \quad 0.53744$

$53 \mathrm{~B}->56 \mathrm{~B} \quad 0.25669$

$54 \mathrm{~B}->56 \mathrm{~B} \quad-0.32605$

$54 \mathrm{~B}->57 \mathrm{~B} \quad-0.12816$

Excited State 15: $1.000-\mathrm{A} \quad 5.6685 \mathrm{eV} 218.72 \mathrm{~nm} \mathrm{f}=0.1308<\mathrm{S} * * 2>=0.000$
$52 \mathrm{~A}->55 \mathrm{~A}$
0.18037
$53 \mathrm{~A} \rightarrow 56 \mathrm{~A} \quad 0.41756$
$54 \mathrm{~A} \rightarrow 57 \mathrm{~A} \quad 0.46745$
$54 \mathrm{~A} \rightarrow 58 \mathrm{~A} \quad-0.15668$
$54 \mathrm{~A} \rightarrow 60 \mathrm{~A} \quad 0.10825$
$52 \mathrm{~B}->55 \mathrm{~B} \quad 0.18037$
$53 \mathrm{~B}->56 \mathrm{~B} \quad 0.41756$
$54 \mathrm{~B}->57 \mathrm{~B} \quad 0.46745$
$54 \mathrm{~B} \rightarrow 58 \mathrm{~B} \quad-0.15668$
$54 \mathrm{~B}->60 \mathrm{~B} \quad 0.10825$

Excited State 26: $1.000-\mathrm{A} \quad 6.4387 \mathrm{eV} 192.56 \mathrm{~nm} \mathrm{f}=0.1056<\mathrm{S} * * 2>=0.000$
$51 \mathrm{~A}->55 \mathrm{~A}$
0.45857
$52 \mathrm{~A}->55 \mathrm{~A}$
0.24550
$53 \mathrm{~A} \rightarrow 57 \mathrm{~A} \quad-0.43832$
$54 \mathrm{~A} \rightarrow 62 \mathrm{~A} \quad 0.10652$
$51 \mathrm{~B}->55 \mathrm{~B} \quad 0.45857$
$52 \mathrm{~B} \rightarrow 55 \mathrm{~B} \quad 0.24550$
$53 \mathrm{~B}->57 \mathrm{~B} \quad-0.43832$
$54 \mathrm{~B}->62 \mathrm{~B} \quad 0.10652$

\section{$\underline{\mathbf{3 d}}$}

Excited State $3: 1.000-\mathrm{A} \quad 3.8363 \mathrm{eV} \quad 323.18 \mathrm{~nm} \mathrm{f}=0.1626<\mathrm{S} * * 2>=0.000$ $51 \mathrm{~A}->53 \mathrm{~A} \quad-0.12800$ 


$$
\begin{array}{lc}
\mathbf{5 2 A}->\text { 53A } & \mathbf{0 . 6 8 3 8 2}(\pi \text { номо- } \pi \text { LUMO, 94\% contribution }) \\
51 \mathrm{~B}->53 \mathrm{~B} & -0.12800 \\
\mathbf{5 2 B}->\text { 53B } & \mathbf{0 . 6 8 3 8 2}(\pi \text { номО- } \pi \text { LUMO, } 94 \% \text { contribution })
\end{array}
$$

Excited State 6: $1.000-\mathrm{A} \quad 4.6745 \mathrm{eV} \quad 265.23 \mathrm{~nm} \mathrm{f}=0.2487<\mathrm{S} * * 2>=0.000$

$$
\begin{array}{ll}
51 \mathrm{~A}->53 \mathrm{~A} & 0.56619 \\
52 \mathrm{~A}>53 \mathrm{~A} & 0.15114 \\
52 \mathrm{~A}->54 \mathrm{~A} & 0.37795 \\
51 \mathrm{~B}->53 \mathrm{~B} & 0.56619 \\
52 \mathrm{~B}->53 \mathrm{~B} & 0.15114 \\
52 \mathrm{~B}->54 \mathrm{~B} & 0.37795
\end{array}
$$

Excited State 9: 1.000-A

$$
\begin{array}{lr}
51 \mathrm{~A} \rightarrow \text { 53A } & -0.29088 \\
52 \mathrm{~A}>54 \mathrm{~A} & 0.44476 \\
52 \mathrm{~A}>55 \mathrm{~A} & 0.42493 \\
52 \mathrm{~A}>58 \mathrm{~A} & 0.10859 \\
51 \mathrm{~B}->53 \mathrm{~B} & -0.29088 \\
52 \mathrm{~B}->54 \mathrm{~B} & 0.44476 \\
52 \mathrm{~B}->55 \mathrm{~B} & 0.42493 \\
52 \mathrm{~B}->58 \mathrm{~B} & 0.10859
\end{array}
$$

$5.1500 \mathrm{eV} 240.75 \mathrm{~nm} \mathrm{f}=0.2647<\mathrm{S}^{* *} 2>=0.000$

Excited State 10: 1.000-A
$51 \mathrm{~A} \rightarrow 53 \mathrm{~A}$
0.24407
$52 \mathrm{~A} \rightarrow 54 \mathrm{~A}$
$-0.31032$
$52 \mathrm{~A} \rightarrow 55 \mathrm{~A}$
0.54495
$52 \mathrm{~A} \rightarrow 56 \mathrm{~A}$
0.14310
$51 \mathrm{~B} \rightarrow 53 \mathrm{~B}$
0.24407
$52 \mathrm{~B}->54 \mathrm{~B}$
$-0.31032$
$52 \mathrm{~B} \rightarrow 55 \mathrm{~B}$
0.54495
$52 \mathrm{~B} \rightarrow 56 \mathrm{~B}$
0.14310

Excited State 12: 1.000-A
$51 \mathrm{~A} \rightarrow 54 \mathrm{~A}$
$-0.29082$
$52 \mathrm{~A} \rightarrow 54 \mathrm{~A}$
0.14703
$52 \mathrm{~A} \rightarrow 56 \mathrm{~A}$
0.55161
$52 \mathrm{~A} \rightarrow 57 \mathrm{~A}$
$-0.23141$
$51 \mathrm{~B}->54 \mathrm{~B}$
$-0.29082$
$52 \mathrm{~B} \rightarrow 54 \mathrm{~B}$
0.14703
$52 \mathrm{~B}->56 \mathrm{~B}$
0.55161
$52 \mathrm{~B}->57 \mathrm{~B}$
$-0.23141$

Excited State 14: 1.000-A

$5.6840 \mathrm{eV} 218.13 \mathrm{~nm} \mathrm{f}=0.1017<\mathrm{S} * * 2>=0.000$
$51 \mathrm{~A} \rightarrow 54 \mathrm{~A}$
0.36957
$52 \mathrm{~A} \rightarrow 54 \mathrm{~A}$
$-0.10275$
$52 \mathrm{~A}->56 \mathrm{~A}$
0.25384
$52 \mathrm{~A} \rightarrow 57 \mathrm{~A}$
0.24431
$52 \mathrm{~A} \rightarrow 58 \mathrm{~A}$
0.45536
$51 \mathrm{~B}->54 \mathrm{~B}$
0.36957
$52 \mathrm{~B} \rightarrow 54 \mathrm{~B}$
$-0.10275$
$52 \mathrm{~B}->56 \mathrm{~B}$
0.25384

$5.6156 \mathrm{eV} 220.78 \mathrm{~nm} \mathrm{f}=0.1305<\mathrm{S}^{* *} 2>=0.000$

$5.2002 \mathrm{eV} 238.42 \mathrm{~nm} \mathrm{f}=0.2112<\mathrm{S} * * 2>=0.000$

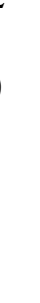




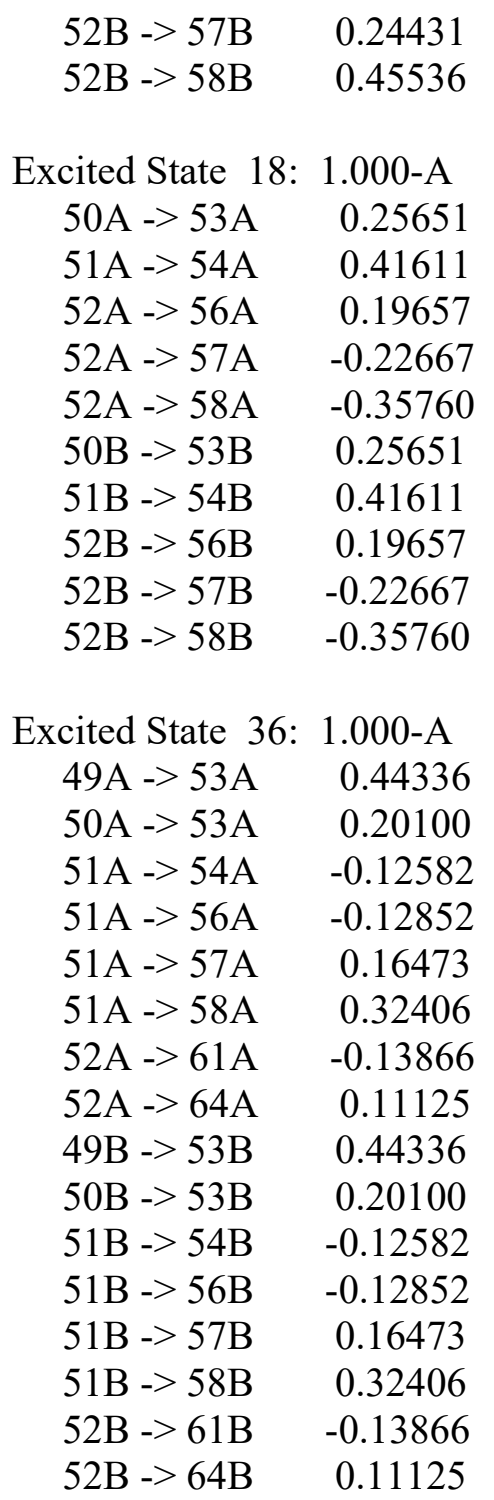

$5.9332 \mathrm{eV} 208.97 \mathrm{~nm} \mathrm{f}=0.1967<\mathrm{S} * * 2>=0.000$

$6.6223 \mathrm{eV} 187.22 \mathrm{~nm} \mathrm{f}=0.2306<\mathrm{S} * * 2>=0.000$
$49 \mathrm{~A}->53 \mathrm{~A}$
0.44336
$51 \mathrm{~A} \rightarrow 54 \mathrm{~A}$
$-0.12582$
$51 \mathrm{~A} \rightarrow 56 \mathrm{~A}$
0.12852
$51 \mathrm{~A}->57 \mathrm{~A}$
0.32406
$52 \mathrm{~A}->61 \mathrm{~A}$
$-0.13866$
$52 \mathrm{~A} \rightarrow>64 \mathrm{~A}$
0.44336
$50 \mathrm{~B}->53 \mathrm{~B} \quad 0.20100$
$51 \mathrm{~B}->54 \mathrm{~B} \quad-0.12582$
$51 \mathrm{~B}->56 \mathrm{~B} \quad-0.12852$
$51 \mathrm{~B}->57 \mathrm{~B} \quad 0.16473$
$51 \mathrm{~B}->58 \mathrm{~B} \quad 0.32406$
$52 \mathrm{~B}->64 \mathrm{~B} \quad 0.11125$

\section{$\underline{3 \mathbf{e}}$}

Excited State $4: 1.000-\mathrm{A} \quad 3.9557 \mathrm{eV} \quad 313.43 \mathrm{~nm} \mathrm{f}=0.2014<\mathrm{S} * * 2>=0.000$ $55 \mathrm{~A} \rightarrow 57 \mathrm{~A} \quad 0.25644$

56A $->$ 57A $\quad \mathbf{0 . 6 4 3 5 3}$ ( $\pi$ НОМО- $\pi$ LUMO, $83 \%$ contribution $)$

$55 \mathrm{~B}->57 \mathrm{~B} \quad 0.25644$

56B -> 57B $\quad 0.64353$ ( $\pi$ номо- $\pi$ LUMO, $83 \%$ contribution $)$

Excited State 6: $1.000-\mathrm{A} \quad 4.4936 \mathrm{eV} \quad 275.91 \mathrm{~nm} \mathrm{f}=0.3797<\mathrm{S}^{* *} 2>=0.000$
$55 \mathrm{~A} \rightarrow 57 \mathrm{~A}$
0.57872
$56 \mathrm{~A} \rightarrow 57 \mathrm{~A} \quad-0.27117$
$56 \mathrm{~A} \rightarrow 58 \mathrm{~A} \quad-0.29364$
$55 \mathrm{~B}->57 \mathrm{~B} \quad 0.57872$
$56 \mathrm{~B}->57 \mathrm{~B} \quad-0.27117$
$56 \mathrm{~B}->58 \mathrm{~B} \quad-0.29364$

Excited State $8: 1.000-\mathrm{A} \quad 5.0241 \mathrm{eV} \quad 246.78 \mathrm{~nm} \mathrm{f}=0.2497<\mathrm{S}^{* *} 2>=0.000$ $55 \mathrm{~A} \rightarrow 57 \mathrm{~A} \quad 0.29763$ 


$$
\begin{array}{lr}
55 \mathrm{~A}->58 \mathrm{~A} & -0.12735 \\
55 \mathrm{~A}->62 \mathrm{~A} & 0.13209 \\
56 \mathrm{~A}->58 \mathrm{~A} & 0.59321 \\
55 \mathrm{~B}->57 \mathrm{~B} & 0.29763 \\
55 \mathrm{~B}->58 \mathrm{~B} & -0.12735 \\
55 \mathrm{~B}->62 \mathrm{~B} & 0.13209 \\
56 \mathrm{~B}->58 \mathrm{~B} & 0.59321
\end{array}
$$

Excited State 12: 1.000-A $55 \mathrm{~A} \rightarrow 58 \mathrm{~A} \quad 0.62645$ $55 \mathrm{~A} \rightarrow 59 \mathrm{~A} \quad 0.10127$ $56 \mathrm{~A} \rightarrow 58 \mathrm{~A} \quad 0.14593$ $56 \mathrm{~A} \rightarrow 60 \mathrm{~A} \quad 0.14262$ $56 \mathrm{~A} \rightarrow 62 \mathrm{~A} \quad-0.10158$ $55 \mathrm{~B}->58 \mathrm{~B} \quad 0.62645$ $55 \mathrm{~B}->59 \mathrm{~B} \quad 0.10127$ $56 \mathrm{~B}->58 \mathrm{~B} \quad 0.14593$ $56 \mathrm{~B}->60 \mathrm{~B} \quad 0.14262$ $56 \mathrm{~B}->62 \mathrm{~B} \quad-0.10158$

Excited State $30: 1.000-\mathrm{A}$
$53 \mathrm{~A} \rightarrow 57 \mathrm{~A}$ $-0.10776$
$54 \mathrm{~A}->57 \mathrm{~A}$ 0.40784
$55 \mathrm{~A} \rightarrow 58 \mathrm{~A}$ $-0.13309$
$55 \mathrm{~A}->62 \mathrm{~A}$ $-0.27664$
$56 \mathrm{~A} \rightarrow 62 \mathrm{~A}$ $-0.31034$
$56 \mathrm{~A}->64 \mathrm{~A}$
0.24106
$53 \mathrm{~B} \rightarrow 57 \mathrm{~B}$
$-0.10776$
$54 \mathrm{~B}->57 \mathrm{~B}$
0.40784
$55 \mathrm{~B}->58 \mathrm{~B}$
$-0.13309$
$55 \mathrm{~B}->62 \mathrm{~B}$
$-0.27664$
$56 \mathrm{~B} \rightarrow 62 \mathrm{~B}$
$-0.31034$
$56 \mathrm{~B}->64 \mathrm{~B}$
0.24106

Excited State $37:$ 1.000-A

$53 \mathrm{~A}->57 \mathrm{~A} \quad-0.15709$

$54 \mathrm{~A} \rightarrow 57 \mathrm{~A} \quad 0.15820$

$54 \mathrm{~A} \rightarrow 58 \mathrm{~A} \quad 0.10743$

$55 \mathrm{~A} \rightarrow 62 \mathrm{~A} \quad 0.50996$

$55 \mathrm{~A} \rightarrow 63 \mathrm{~A} \quad 0.10089$

$55 \mathrm{~A} \rightarrow 64 \mathrm{~A} \quad 0.19270$

$55 \mathrm{~A}->65 \mathrm{~A} \quad 0.12394$

$55 \mathrm{~A} \rightarrow 66 \mathrm{~A} \quad-0.13423$

$56 \mathrm{~A} \rightarrow 66 \mathrm{~A} \quad 0.22175$

$53 \mathrm{~B}->57 \mathrm{~B} \quad-0.15709$

$54 \mathrm{~B}->57 \mathrm{~B} \quad 0.15820$

$54 \mathrm{~B}->58 \mathrm{~B} \quad 0.10743$

$55 \mathrm{~B}->62 \mathrm{~B} \quad 0.50996$

$55 \mathrm{~B}->63 \mathrm{~B} \quad 0.10089$

$55 \mathrm{~B}->64 \mathrm{~B} \quad 0.19270$

$55 \mathrm{~B}->65 \mathrm{~B} \quad 0.12394$
$5.5040 \mathrm{eV} 225.26 \mathrm{~nm} \mathrm{f}=0.2813<\mathrm{S} * * 2>=0.000$

$6.2839 \mathrm{eV} 197.30 \mathrm{~nm} \mathrm{f}=0.2689<\mathrm{S} * * 2>=0.000$

$6.4995 \mathrm{eV} 190.76 \mathrm{~nm} \mathrm{f}=0.1388<\mathrm{S} * * 2>=0.000$ 
$55 \mathrm{~B}->66 \mathrm{~B} \quad-0.13423$

$56 \mathrm{~B}->66 \mathrm{~B} \quad 0.22175$

$\underline{\mathbf{3 f}}$

Excited State $3: 1.000-\mathrm{A} \quad 3.5432 \mathrm{eV} \quad 349.92 \mathrm{~nm} \mathrm{f}=0.1730<\mathrm{S}^{* *} 2>=0.000$

54A $\rightarrow$ 55A $\quad \mathbf{0 . 6 9 1 6 2}(\pi$ HOMO- $\pi$ LUMO, $96 \%$ contribution $)$

54B $->55 B \quad 0.69162$ ( $\pi$ номо- $\pi$ LUMO, $96 \%$ contribution $)$

Excited State 6: $1.000-\mathrm{A} \quad 4.5537 \mathrm{eV} \quad 272.27 \mathrm{~nm} \mathrm{f}=0.4368<\mathrm{S}^{* *} 2>=0.000$

$53 \mathrm{~A} \rightarrow 55 \mathrm{~A} \quad 0.63501$

$54 \mathrm{~A} \rightarrow 55 \mathrm{~A} \quad 0.11278$

$54 \mathrm{~A} \rightarrow 56 \mathrm{~A} \quad-0.26468$

$53 \mathrm{~B}->55 \mathrm{~B} \quad 0.63501$

$54 \mathrm{~B}->55 \mathrm{~B} \quad 0.11278$

$54 \mathrm{~B}->56 \mathrm{~B} \quad-0.26468$

Excited State 9: $1.000-\mathrm{A} \quad 5.0792 \mathrm{eV} \quad 244.10 \mathrm{~nm} \mathrm{f}=0.1484<\mathrm{S}^{* *} 2>=0.000$

$53 \mathrm{~A} \rightarrow 55 \mathrm{~A} \quad 0.19534$

$54 \mathrm{~A} \rightarrow 56 \mathrm{~A} \quad 0.54247$

$54 \mathrm{~A} \rightarrow 57 \mathrm{~A} \quad 0.37892$

$53 \mathrm{~B}->55 \mathrm{~B} \quad 0.19534$

$54 \mathrm{~B}->56 \mathrm{~B} \quad 0.54247$

$54 \mathrm{~B}->57 \mathrm{~B} \quad 0.37892$

Excited State 12: $1.000-\mathrm{A} \quad 5.4559 \mathrm{eV} \quad 227.25 \mathrm{~nm} \mathrm{f}=0.5638<\mathrm{S} * * 2>=0.000$ $52 \mathrm{~A} \rightarrow 55 \mathrm{~A} \quad-0.10472$

$53 \mathrm{~A} \rightarrow 55 \mathrm{~A} \quad-0.18967$

$54 \mathrm{~A} \rightarrow 56 \mathrm{~A} \quad-0.32837$

$54 \mathrm{~A} \rightarrow 57 \mathrm{~A} \quad 0.54596$

$54 \mathrm{~A} \rightarrow 58 \mathrm{~A} \quad 0.15600$

$52 \mathrm{~B}->55 \mathrm{~B} \quad-0.10472$

$53 \mathrm{~B}->55 \mathrm{~B} \quad-0.18967$

$54 \mathrm{~B}->56 \mathrm{~B} \quad-0.32837$

$54 \mathrm{~B}->57 \mathrm{~B} \quad 0.54596$

$54 \mathrm{~B}->58 \mathrm{~B} \quad 0.15600$

Excited State 26: 1.000-A

$50 \mathrm{~A} \rightarrow 55 \mathrm{~A} \quad-0.13171$

$51 \mathrm{~A} \rightarrow 55 \mathrm{~A} \quad 0.43721$

$52 \mathrm{~A} \rightarrow 55 \mathrm{~A} \quad-0.14460$

$53 \mathrm{~A} \rightarrow 56 \mathrm{~A} \quad 0.32329$

$53 \mathrm{~A} \rightarrow 57 \mathrm{~A} \quad-0.34569$

$50 \mathrm{~B}->55 \mathrm{~B} \quad-0.13171$

$51 \mathrm{~B}->55 \mathrm{~B} \quad 0.43721$

$52 \mathrm{~B}->55 \mathrm{~B} \quad-0.14460$

$53 \mathrm{~B}->56 \mathrm{~B} \quad 0.32329$

$53 \mathrm{~B}->57 \mathrm{~B} \quad-0.34569$

3g

Excited State $3: 1.000-\mathrm{A} \quad 3.4453 \mathrm{eV} \quad 359.86 \mathrm{~nm} \mathrm{f}=0.1523<\mathrm{S}^{*} * 2>=0.000$ 


$$
\begin{array}{ll}
\text { 63A }>\text { 64A } & \mathbf{0 . 6 9 4 6 0}(\pi \text { нОмО- } \pi \text { LUMO, 97\% contribution }) \\
\text { 63B }->\text { 64B } & \mathbf{0 . 6 9 4 6 0}(\pi \text { номо- } \pi \text { LUMO, } 97 \% \text { contribution })
\end{array}
$$

Excited State 7: 1.000-A
$62 \mathrm{~A} \rightarrow$ 64A
0.66025
$63 \mathrm{~A} \rightarrow 65 \mathrm{~A}$
0.13646
$63 \mathrm{~A} \rightarrow 66 \mathrm{~A}$
0.16868
$62 \mathrm{~B} \rightarrow 64 \mathrm{~B}$
0.66025
$63 \mathrm{~B} \rightarrow$ $>65 \mathrm{~B}$
0.13646
$63 \mathrm{~B} \rightarrow>66 \mathrm{~B}$
0.16868

Excited State 14: 1.000-A
$62 \mathrm{~A} \rightarrow 64 \mathrm{~A}$
$-0.19053$
$63 \mathrm{~A} \rightarrow 65 \mathrm{~A}$
0.23870
$63 \mathrm{~A}->66 \mathrm{~A}$
0.56457
$63 \mathrm{~A}->67 \mathrm{~A}$
0.26058
$62 \mathrm{~B} \rightarrow>64 \mathrm{~B}$
$-0.19053$
$63 \mathrm{~B} \rightarrow>65 \mathrm{~B}$
0.23870
$63 \mathrm{~B} \rightarrow 66 \mathrm{~B}$
0.56457
$63 \mathrm{~B}->67 \mathrm{~B}$
0.26058

$4.4699 \mathrm{eV} 277.38 \mathrm{~nm} \mathrm{f}=0.4842<\mathrm{S} * * 2>=0.000$

Excited State 15: 1.000-A

$63 \mathrm{~A}->66 \mathrm{~A} \quad-0.22904$

$63 \mathrm{~A} \rightarrow 67 \mathrm{~A} \quad 0.62419$

$63 \mathrm{~A}->68 \mathrm{~A} \quad-0.15356$

$63 \mathrm{~B}->66 \mathrm{~B} \quad-0.22904$

$63 \mathrm{~B}->67 \mathrm{~B} \quad 0.62419$

$63 \mathrm{~B}->68 \mathrm{~B} \quad-0.15356$

Excited State 26: 1.000-A

$58 \mathrm{~A} \rightarrow 64 \mathrm{~A} \quad-0.24788$

$59 \mathrm{~A} \rightarrow 64 \mathrm{~A} \quad 0.27089$

$62 \mathrm{~A} \rightarrow 66 \mathrm{~A} \quad 0.41897$

$62 \mathrm{~A} \rightarrow 67 \mathrm{~A} \quad 0.11578$

$63 \mathrm{~A} \rightarrow 70 \mathrm{~A} \quad 0.24624$

$63 \mathrm{~A} \rightarrow 71 \mathrm{~A} \quad-0.11826$

$63 \mathrm{~A} \rightarrow 74 \mathrm{~A} \quad 0.11670$

$63 \mathrm{~A} \rightarrow 75 \mathrm{~A} \quad 0.14532$

$58 \mathrm{~B}->64 \mathrm{~B} \quad-0.24788$

$59 \mathrm{~B}->64 \mathrm{~B} \quad 0.27089$

$62 \mathrm{~B}->66 \mathrm{~B} \quad 0.41897$

$62 \mathrm{~B}->67 \mathrm{~B} \quad 0.11578$

$63 \mathrm{~B}->70 \mathrm{~B} \quad 0.24624$

$63 \mathrm{~B}->71 \mathrm{~B} \quad-0.11826$

$63 \mathrm{~B}->74 \mathrm{~B} \quad 0.11670$

$63 \mathrm{~B}->75 \mathrm{~B} \quad 0.14532$
$5.3444 \mathrm{eV} 231.99 \mathrm{~nm} \mathrm{f}=0.4828<\mathrm{S} * * 2>=0.000$

$5.3844 \mathrm{eV} 230.26 \mathrm{~nm} \mathrm{f}=0.1223<\mathrm{S} * * 2>=0.000$

$6.2888 \mathrm{eV} 197.15 \mathrm{~nm} \mathrm{f}=0.1722<\mathrm{S} * * 2>=0.000$ 


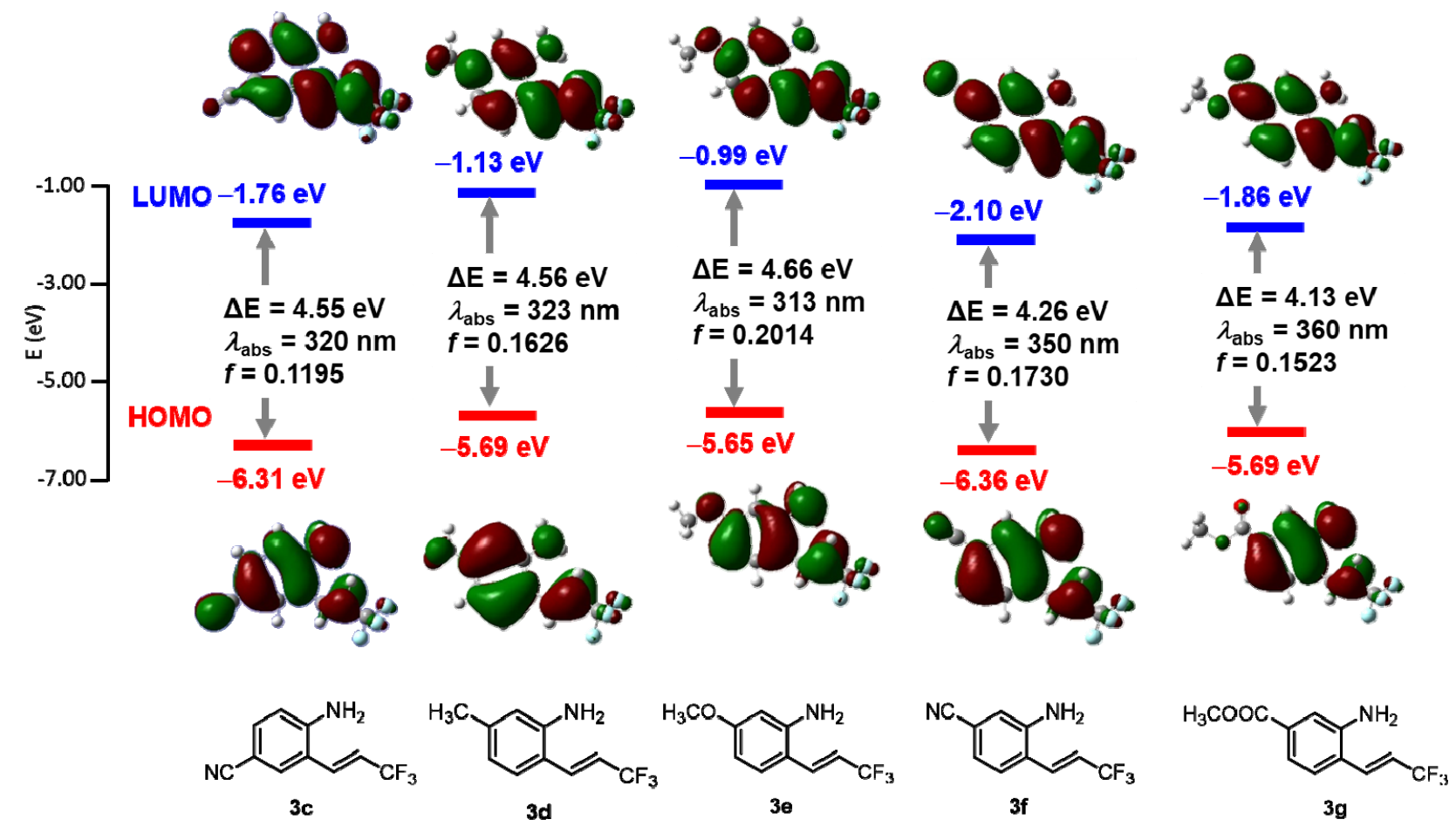

Figure S1. Frontier Molecular Orbitals and Energy Levels of 3c-g Calculated at the RB3LYP/631G(d,p) Level of Theory. Excitation Energies Calculated by TD-DFT calculations at the UB3LYP/6-31+G(d,p) Level of Theory. The $f$ values Refer to the Oscillator Strength.

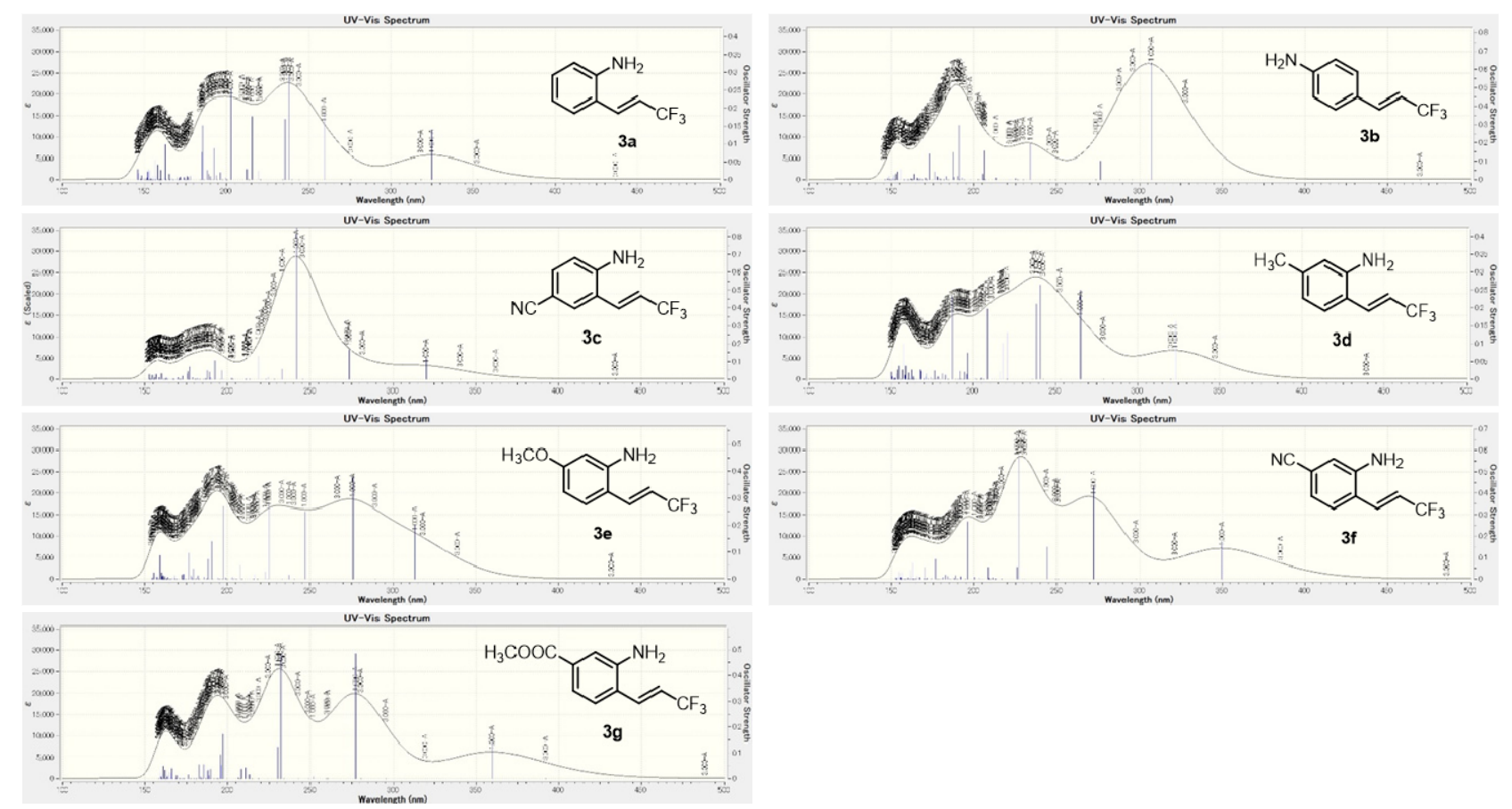

Figure S2. Electronic Transition and Simulated Absorption Spectra of 3a-g Obtained by TD-DFT calculations at the UB3LYP/6-31+G(d,p) Level of Theory. 


\section{Reference}

${ }^{1}$ Gaussian 16, Revision B.01, M. J. Frisch, G. W. Trucks, H. B. Schlegel, G. E. Scuseria, M. A. Robb, J. R. Cheeseman, G. Scalmani, V. Barone, G. A. Petersson, H. Nakatsuji, X. Li, M. Caricato, A. V. Marenich, J. Bloino, B. G. Janesko, R. Gomperts, B. Mennucci, H. P. Hratchian, J. V. Ortiz, A. F. Izmaylov, J. L. Sonnenberg, D. Williams-Young, F. Ding, F. Lipparini, F. Egidi, J. Goings, B. Peng, A. Petrone, T. Henderson, D. Ranasinghe, V. G. Zakrzewski, J. Gao, N. Rega, G. Zheng, W. Liang, M. Hada, M. Ehara, K. Toyota, R. Fukuda, J. Hasegawa, M. Ishida, T. Nakajima, Y. Honda, O. Kitao, H. Nakai, T. Vreven, K. Throssell, J. A. Montgomery, Jr., J. E. Peralta, F. Ogliaro, M. J. Bearpark, J. J. Heyd, E. N. Brothers, K. N. Kudin, V. N. Staroverov, T. A. Keith, R. Kobayashi, J. Normand, K. Raghavachari, A. P. Rendell, J. C. Burant, S. S. Iyengar, J. Tomasi, M. Cossi, J. M. Millam, M. Klene, C. Adamo, R. Cammi, J. W. Ochterski, R. L. Martin, K. Morokuma, O. Farkas, J. B. Foresman, and D. J. Fox, Gaussian, Inc., Wallingford CT, 2016. 
Table S8. FWHM of 3a and 3c to $3 \mathrm{~g}$.

\begin{tabular}{lcccc}
\hline & $\mathrm{FWHM}^{\mathrm{a}} / \mathrm{nm}$ & $\mathrm{FWHM}^{\mathrm{b}} / \mathrm{nm}$ & $\mathrm{FWHM}^{\mathrm{c}} / \mathrm{nm}$ & $\mathrm{FWHM}^{\mathrm{d}} / \mathrm{nm}$ \\
\hline 3a & 56 & - & 58 & 74 \\
$\mathbf{3 c}$ & 60 & - & 50 & 64 \\
$\mathbf{3 d}$ & 53 & 52 & 56 & 73 \\
3e & 50 & - & 53 & 67 \\
$\mathbf{3 f}$ & 59 & 61 & 53 & 64 \\
$\mathbf{3 g}$ & 60 & 61 & 52 & 60
\end{tabular}

${ }^{a}$ Electronic absorption in THF; ${ }^{b}$ Electronic absorption in aqueous solution $\left(\mathrm{H}_{2} \mathrm{O}: \mathrm{DMSO}=9: 1\right)$; ${ }^{c}$ Fluorescence spectra in THF; ${ }^{d}$ Fluorescence spectra in aqueous solution $\left(\mathrm{H}_{2} \mathrm{O}: \mathrm{DMSO}=9: 1\right)$.

Measurement of enzyme activity using H-Ala-3f as a substrate. The experimental procedure was the same as "Enzyme reaction of H-Ala-3f with APN" in the experimental section. Figure S3 shows the hydrolysis velocity of H-Ala-3f $(0.1 \mathrm{mM})$ by APN. The linear increase in velocity was observed between 1 and $10 \mathrm{ng}$ APN. This indicates the degradation of H-Ala-3f is depend on the APN concentration. Accordingly, APN activity can be measured by this procedure.

Figure S3. Dose dependence on the hydrolysis of H-Ala-3f.

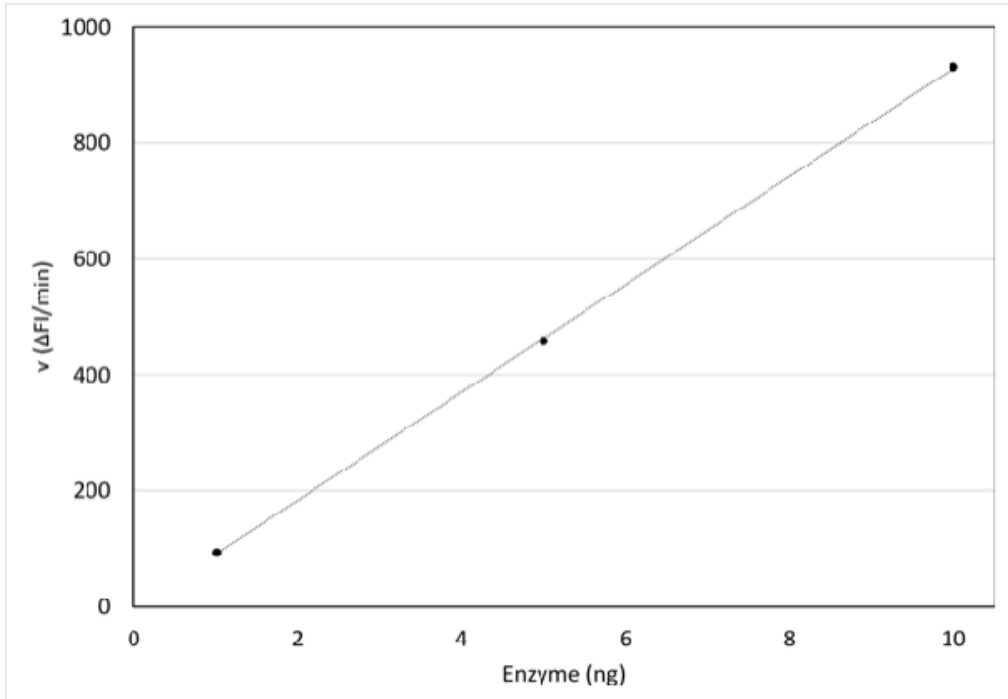


(E)-2-(3,3,3-trifluoroprop-1-enyl)aniline (3a)
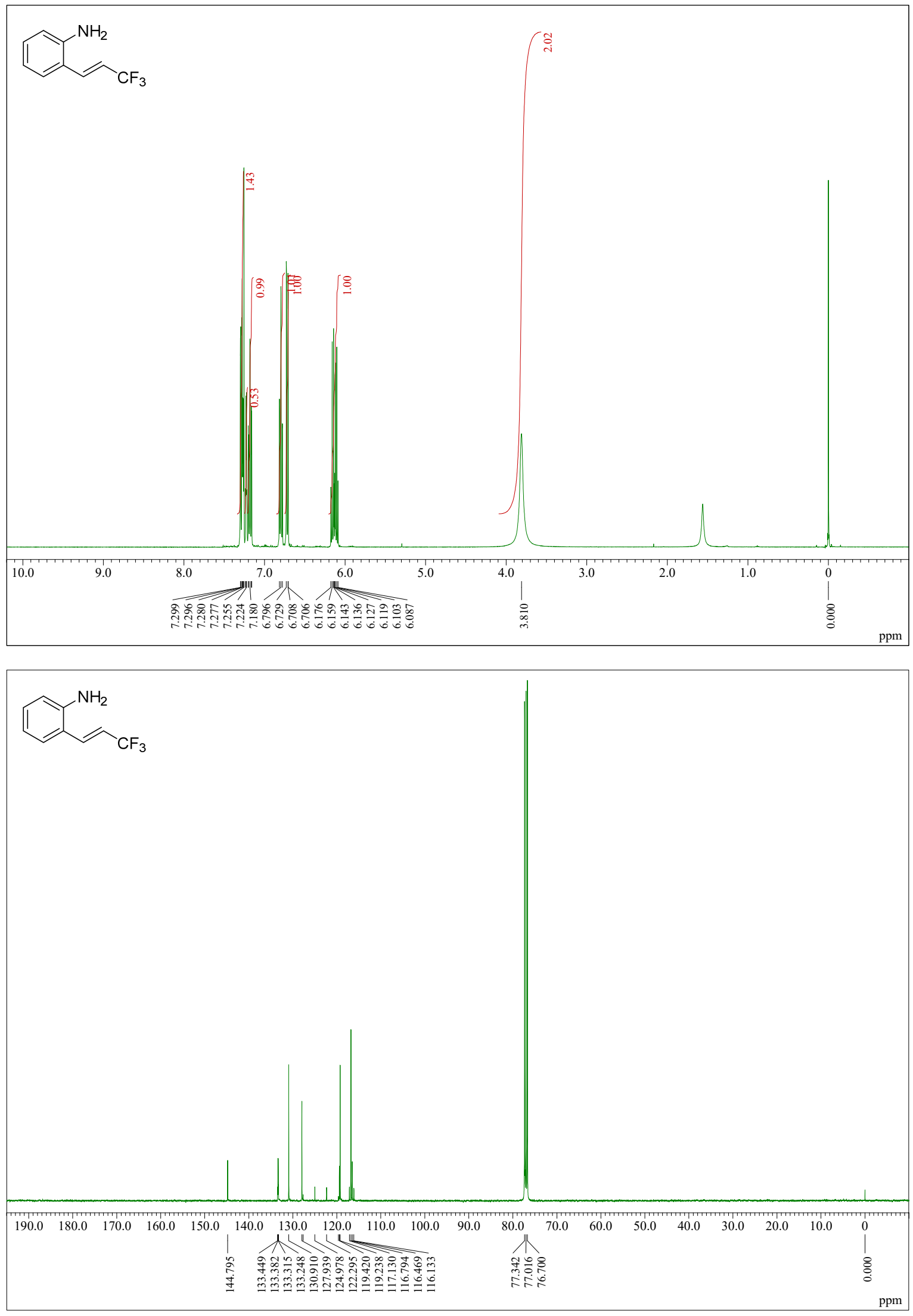
(E)-4-(3,3,3-trifluoroprop-1-enyl)aniline (3b)

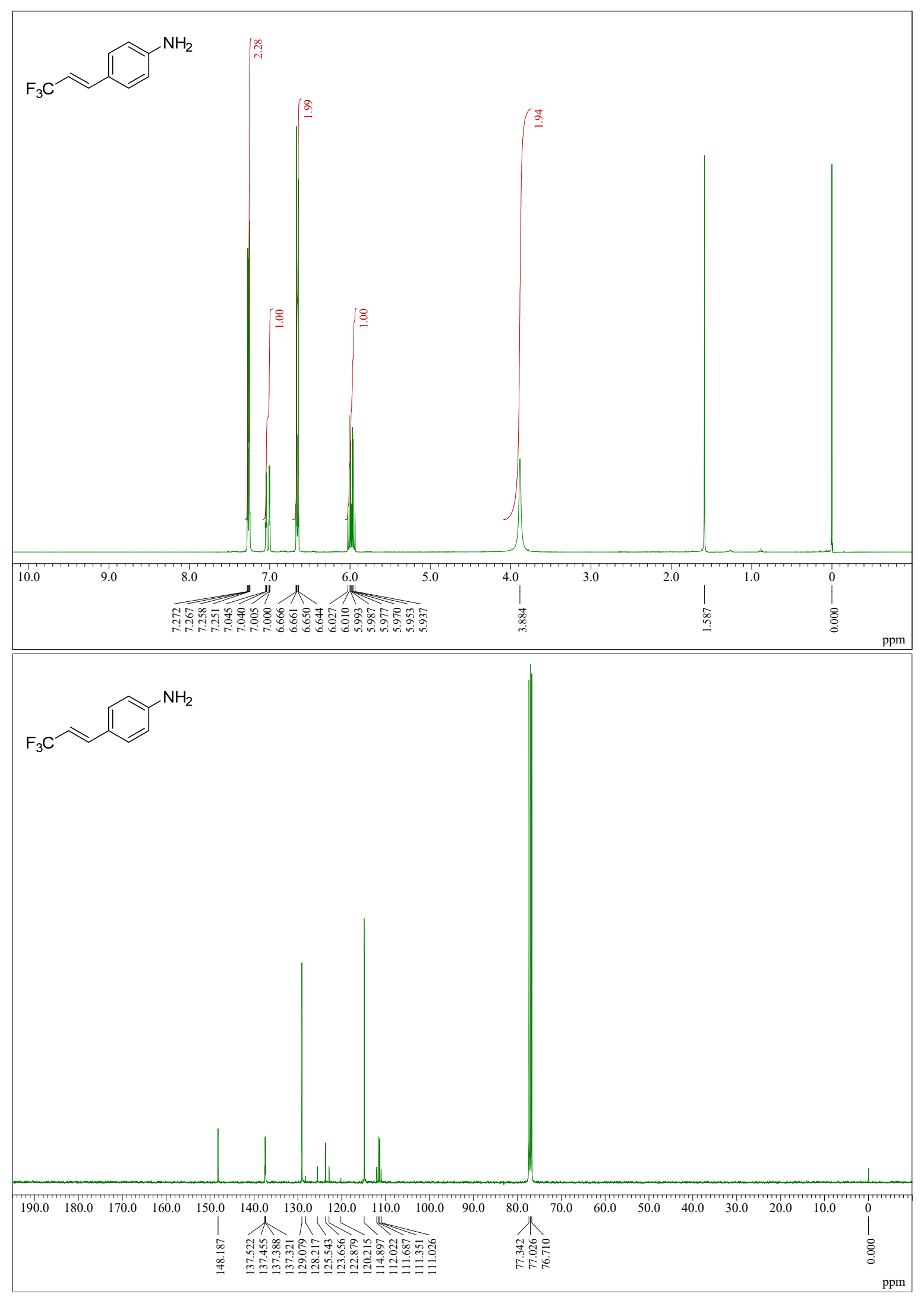

S22 
(E)-4-amino-3-(3,3,3-trifluoroprop-1-enyl)benzonitrile (3c)
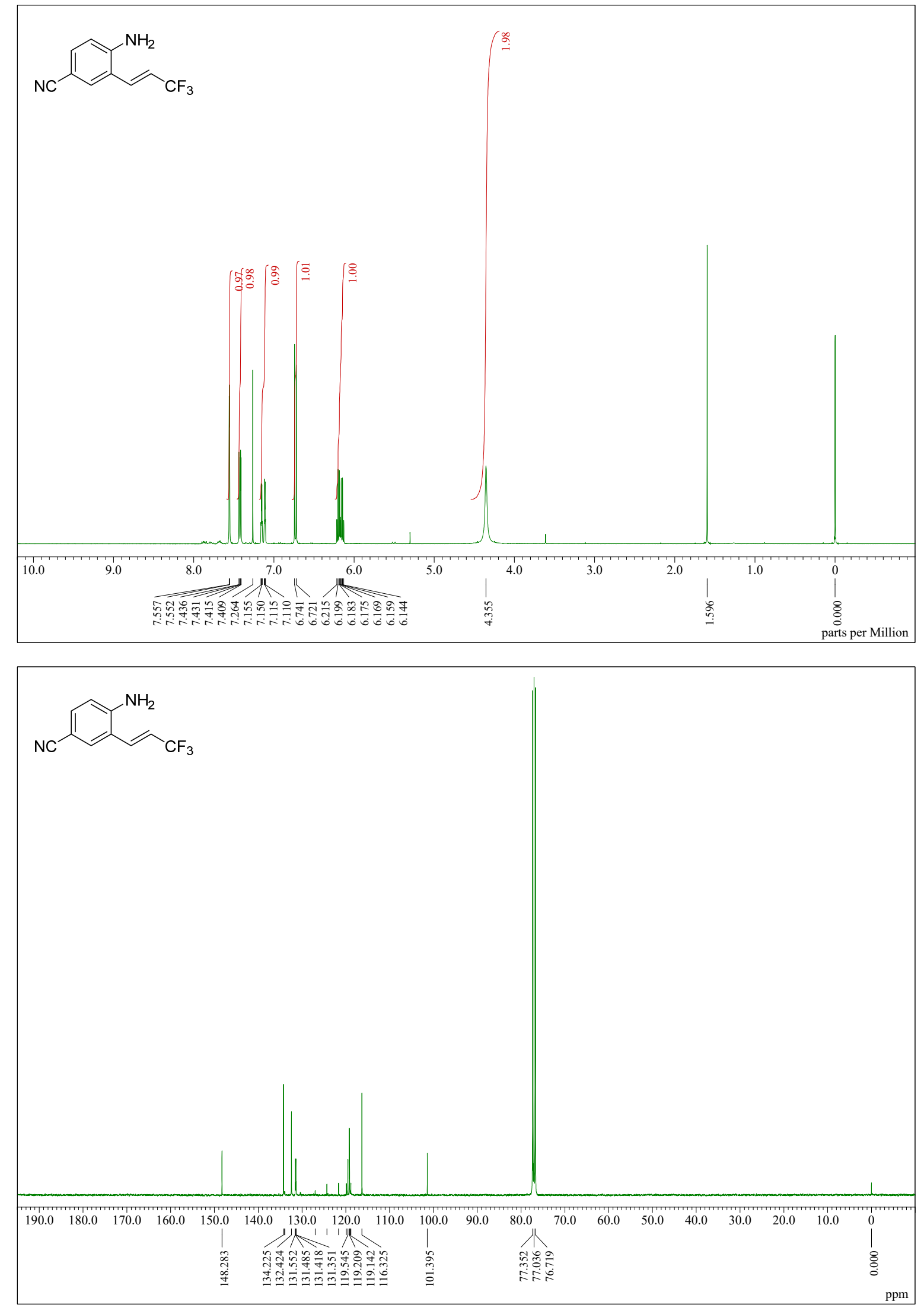
(E)-5-Methyl-2-(3,3,3-trifluoroprop-1-enyl)aniline (3d)

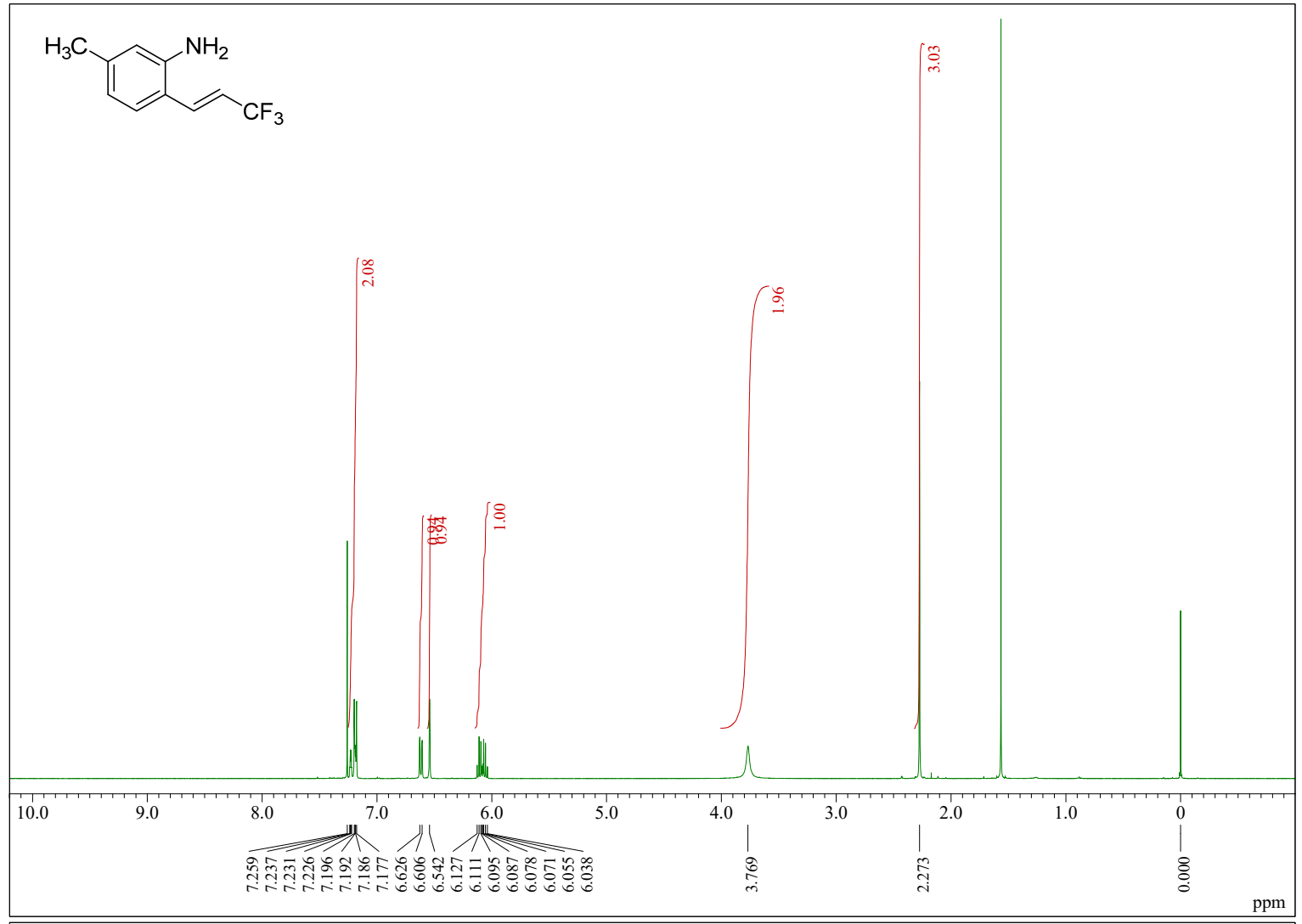<smiles>Cc1ccc(/C=C/C(F)(F)F)c(N)c1</smiles>

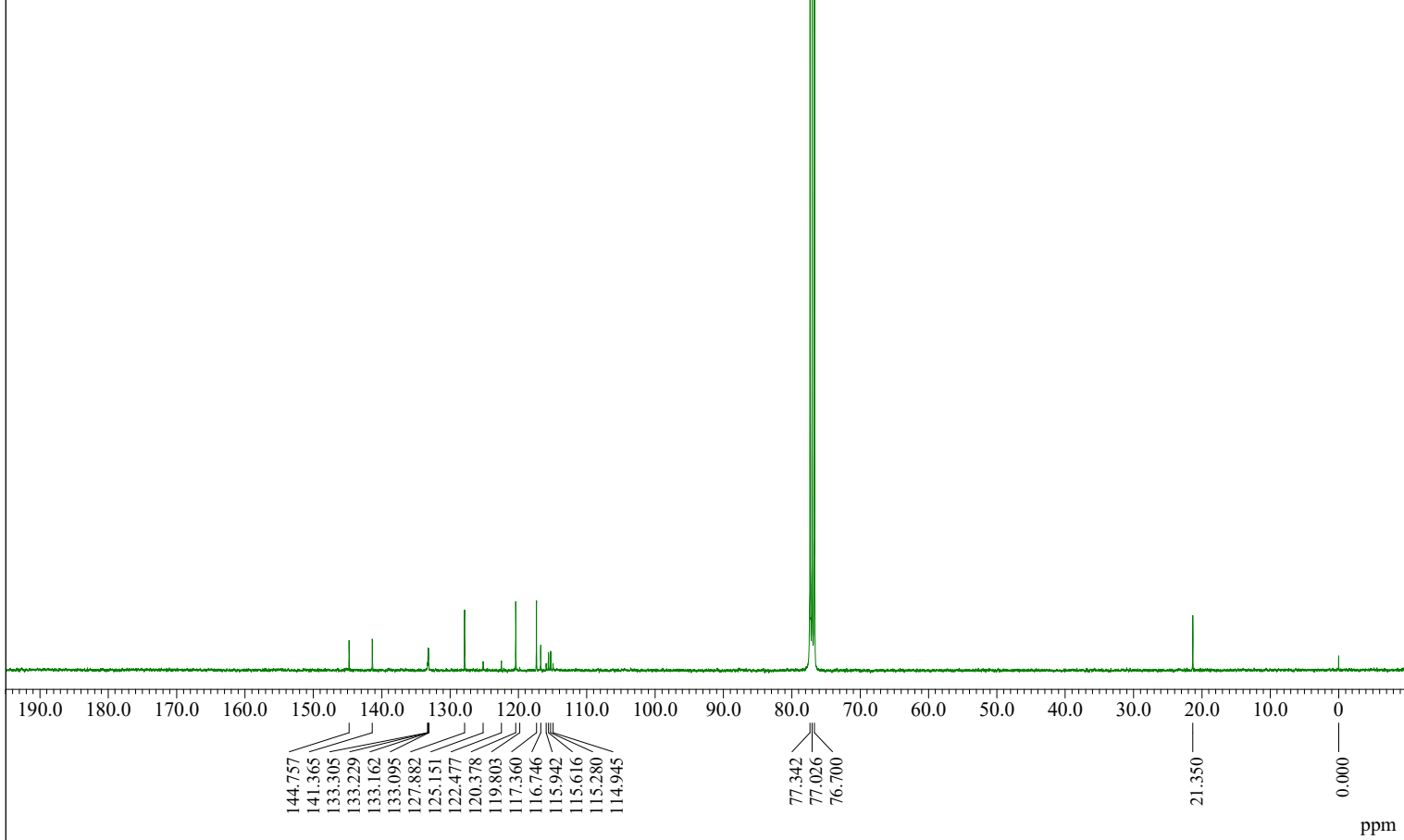


(E)-5-Methoxy-2-(3,3,3-trifluoroprop-1-enyl)aniline (3e)

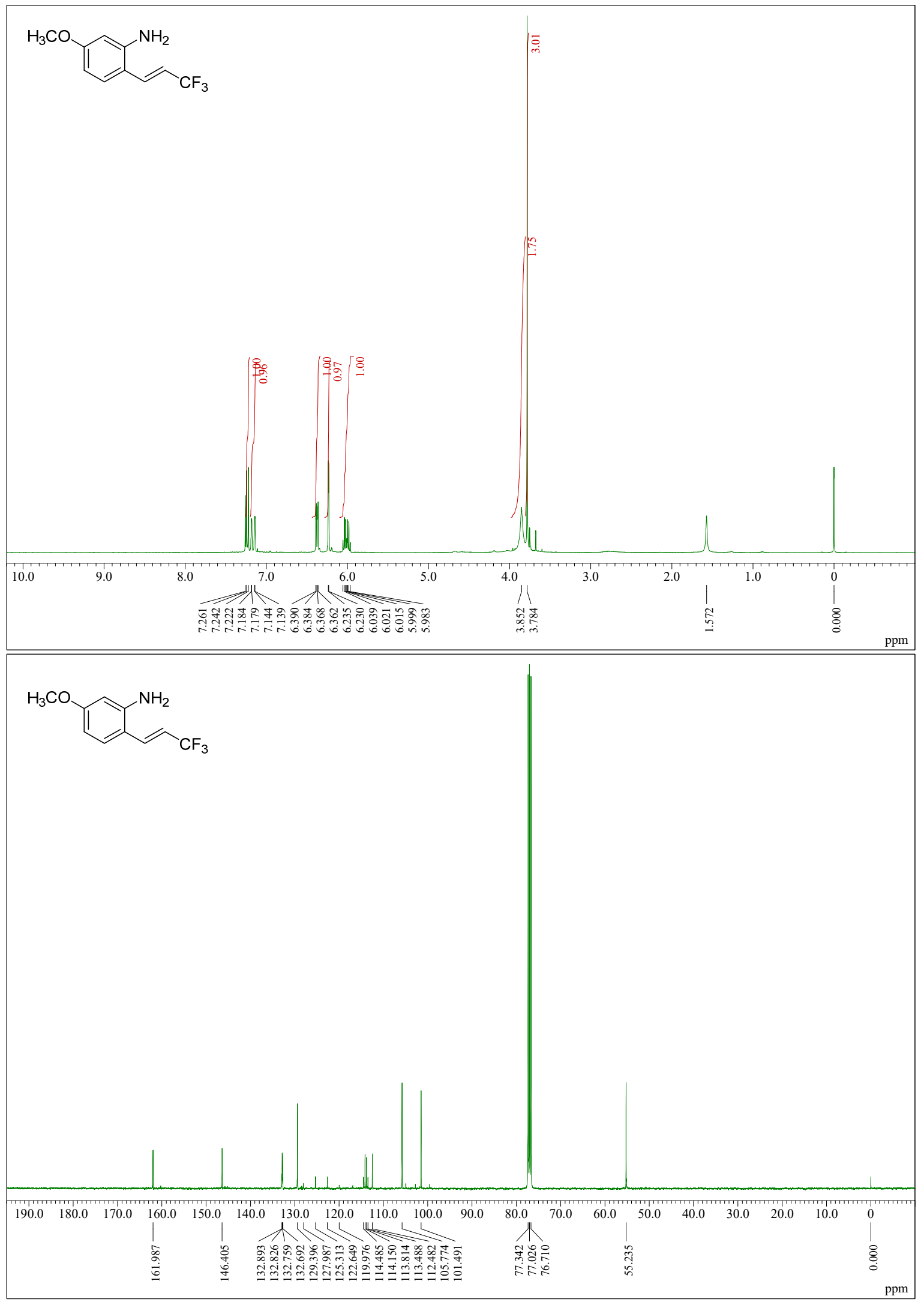

S25 
(E)-3-Amino-4-(3,3,3-trifluoroprop-1-enyl)benzonitrile (3f)

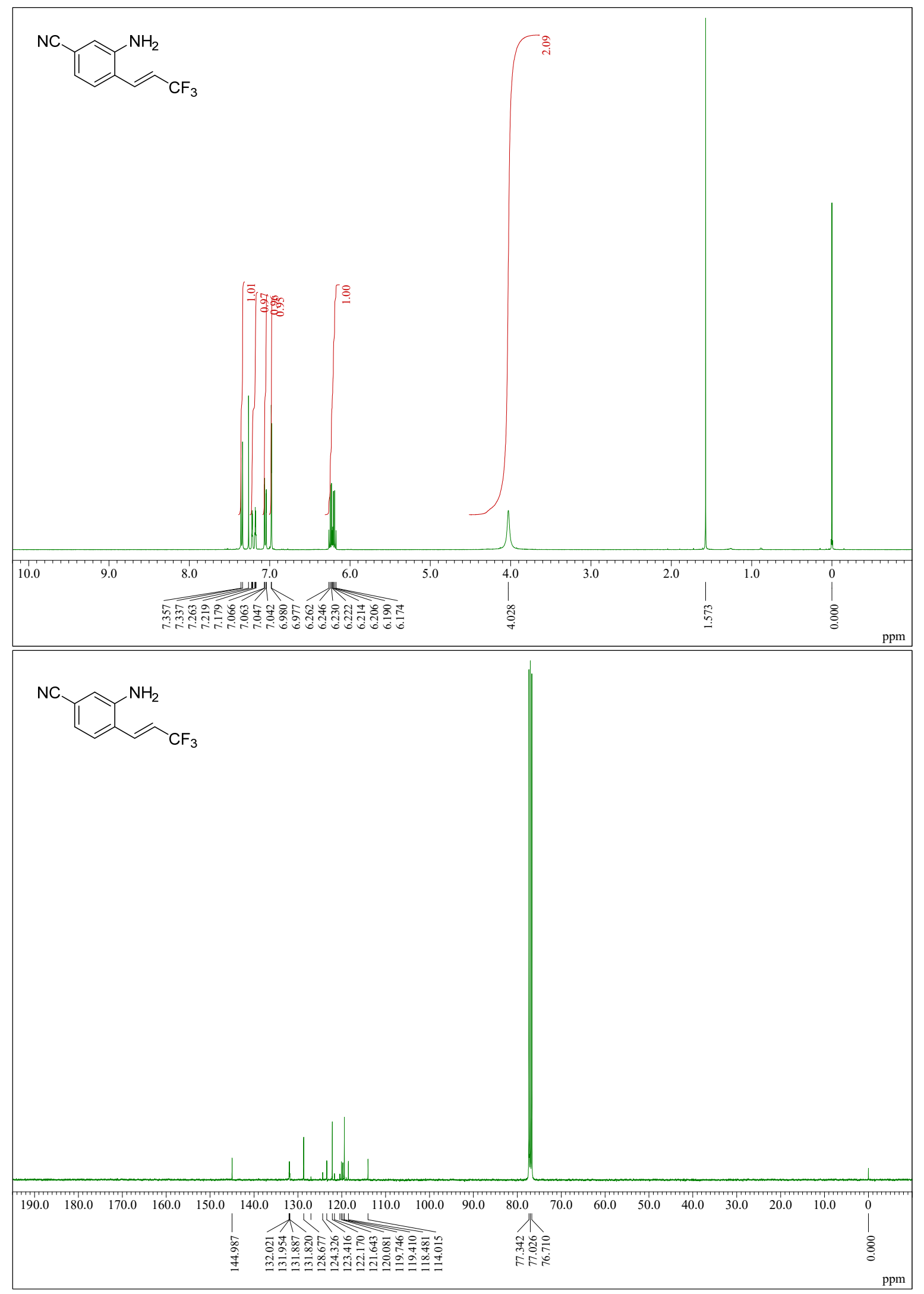

S26 
(E)-Methyl 3-amino-4-(3,3,3-trifluoroprop-1-enyl)benzoate (3g)

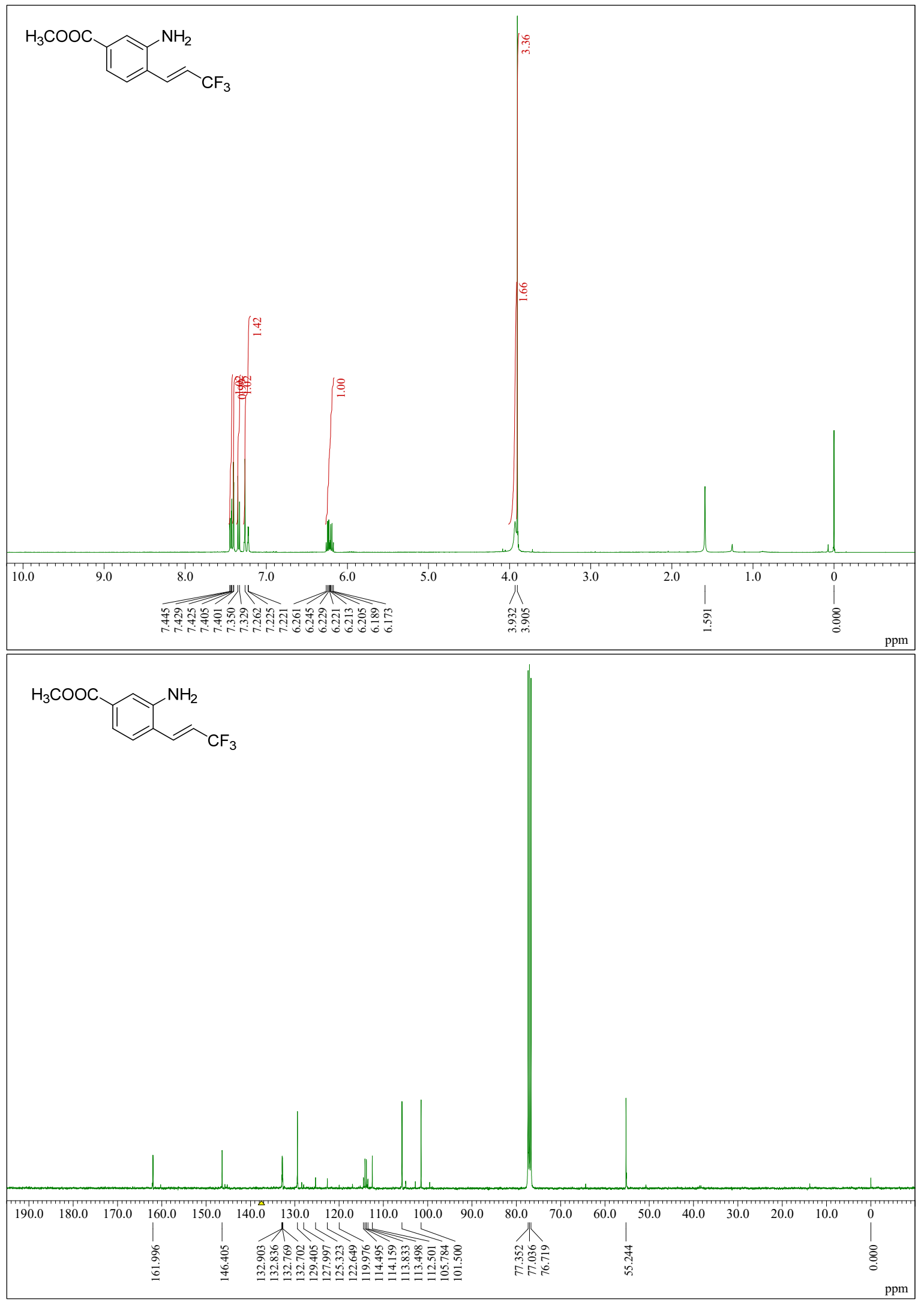

S27 
H-Ala-3f
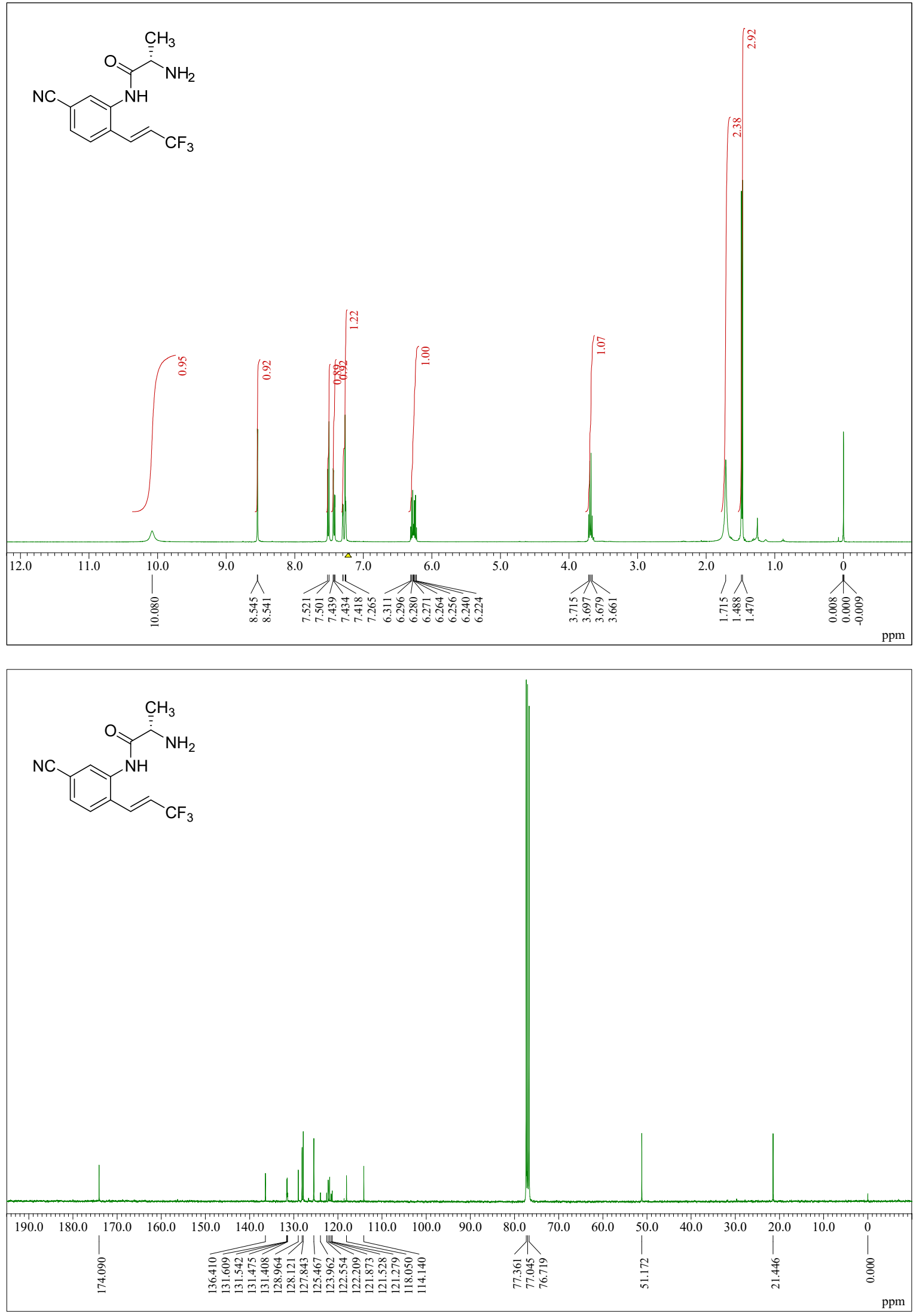